\title{
Planetary nebulae with Wolf-Rayet-type central stars - I. The case of the high-excitation NGC 2371
}

\author{
V. M. A. Gómez-González ${ }^{1 \star}$, J. A. Toalá ${ }^{1}$, M. A. Guerrero² ${ }^{2}$ H. Todt ${ }^{3}$, L. Sabin ${ }^{4}$, \\ G. Ramos-Larios ${ }^{5}$ and Y. D. Mayya ${ }^{6}$ \\ ${ }^{1}$ Instituto de Radioastronomía y Astrofísica (IRyA), UNAM Campus Morelia, Apartado postal 3-72, 58090 Morelia, Michoacán, Mexico \\ ${ }^{2}$ Instituto de Astrofísica de Andalucía (IAA-CSIC), Glorieta de la Astronomía S/N, 18008 Granada, Spain \\ ${ }^{3}$ Institute for Physics and Astronomy, Universität Potsdam, Karl-Liebknecht-Str. 24/25, D-14476 Potsdam, Germany \\ ${ }^{4}$ Instituto de Astronomía, UNAM, Apdo. Postal 877, Ensenada 22860, B.C., Mexico \\ ${ }^{5}$ Instituto de Astronomía y Meteorología, CUCEI, Universidad de Guadalajara, Av. Vallarta 2602, Arcos Vallarta, 44130 Guadalajara, Mexico \\ ${ }^{6}$ Instituto Nacional de Astrofísica, Óptica y Electrónica, Luis Enrique Erro 1, Tonantzintla 72840, Puebla, Mexico
}

1 June 2020

\begin{abstract}
We present the analysis of the planetary nebula (PN) NGC 2371 around the [Wolf-Rayet] ([WR]) star WD 0722+295. Our Isaac Newton Telescope (INT) Intermediate Dispersion Spectrograph (IDS) spectra, in conjunction with archival optical and UV images, unveil in unprecedented detail the high-ionisation of NGC 2371. The nebula has an apparent multipolar morphology, with two pairs of lobes protruding from a barrel-like central cavity, a pair of dense low-ionisation knots misaligned with the symmetry axis embedded within the central cavity, and a high excitation halo mainly detected in He II. The abundances from the barrel-like central cavity and dense knots agree with abundance determinations for other PNe with [WR]-type CSPNe. We suggest that the densest knots inside NGC 2371 are the oldest structures, remnant of a dense equatorial structure, whilst the main nebular shell and outer lobes resulted from a latter ejection that ended the stellar evolution. The analysis of position-velocity diagrams produced from our high-quality spectra suggests that NGC 2371 has a bipolar shape with each lobe presenting a double-structure protruding from a barrel-like central region. The analysis of the spectra of WD $0722+295$ results in similar stellar parameters as previously reported. We corroborate that the spectral sub-type corresponds with a [WO1] type.
\end{abstract}

Key words: stars: evolution — stars: winds, outflows - (ISM:) planetary nebulae: general - (ISM:) planetary nebulae: individual: NGC 2371

\section{INTRODUCTION}

Planetary nebulae $(\mathrm{PNe})$ represent a short-lived configuration of the circumstellar medium of evolved low- and intermediate-mass stars $\left(1 \mathrm{M}_{\odot} \lesssim M_{\mathrm{i}} \lesssim 8 \mathrm{M}_{\odot}\right)$. The interacting stellar winds model of formation predicts that low- and intermediate-mass stars evolve through the asymptotic giant branch (AGB) phase producing a dense and slow wind, which eventually will be swept and compressed by the future fast stellar wind of the post-AGB star and subsequently ionised by the newly developed UV flux (see Kwok et al. 1978; Balick 1987). The wide variety of PN morphologies (round, bipolar, multipolar, irregular, etc; see Sahai et al. 2011) has led to the suggestion that several agents might contribute to the PN shaping, including binarity (or multiple systems; Akashi \& Soker 2017), jets and magnetic fields (see the review by Zijlstra 2015).

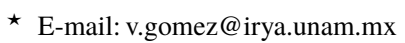

Central stars of PNe (CSPNe) commonly show the presence of hydrogen in their surfaces. However, about $10 \%$ of CSPNe do not exhibit hydrogen features, i.e., they are hydrogen-deficient, rather displaying emission features in their spectra similar to those of the massive Wolf-Rayet (WR) stars of the carbon sequence (see Acker \& Neiner 2003; Górny 2001, and references therein). Accordingly, they are classified in the same way as WR stars, just using square brackets to denote that they are CSPNe instead (Tylenda et al. 1993; Crowther et al. 1998; Acker \& Neiner 2003). It is interesting to note that only a few PNe have been reported to harbor hydrogen-poor [WR] CSPN of the nitrogen sequence: the transitional [WN/WC]type PB 8 (Todt et al. 2010) and the [WN]-type CSPNe of Abell 48 (Todt et al. 2013a) and IC 4663 (Miszalski et al. 2012).

In general, abundance determination of PNe with [WR]-type CSPNe do not present major differences compared to those classical PNe with hydrogen-rich CSPNe (e.g., Peña et al. 1998, 2001). Hydrogen-deficient material ejected inside the old, hydrogen-rich 

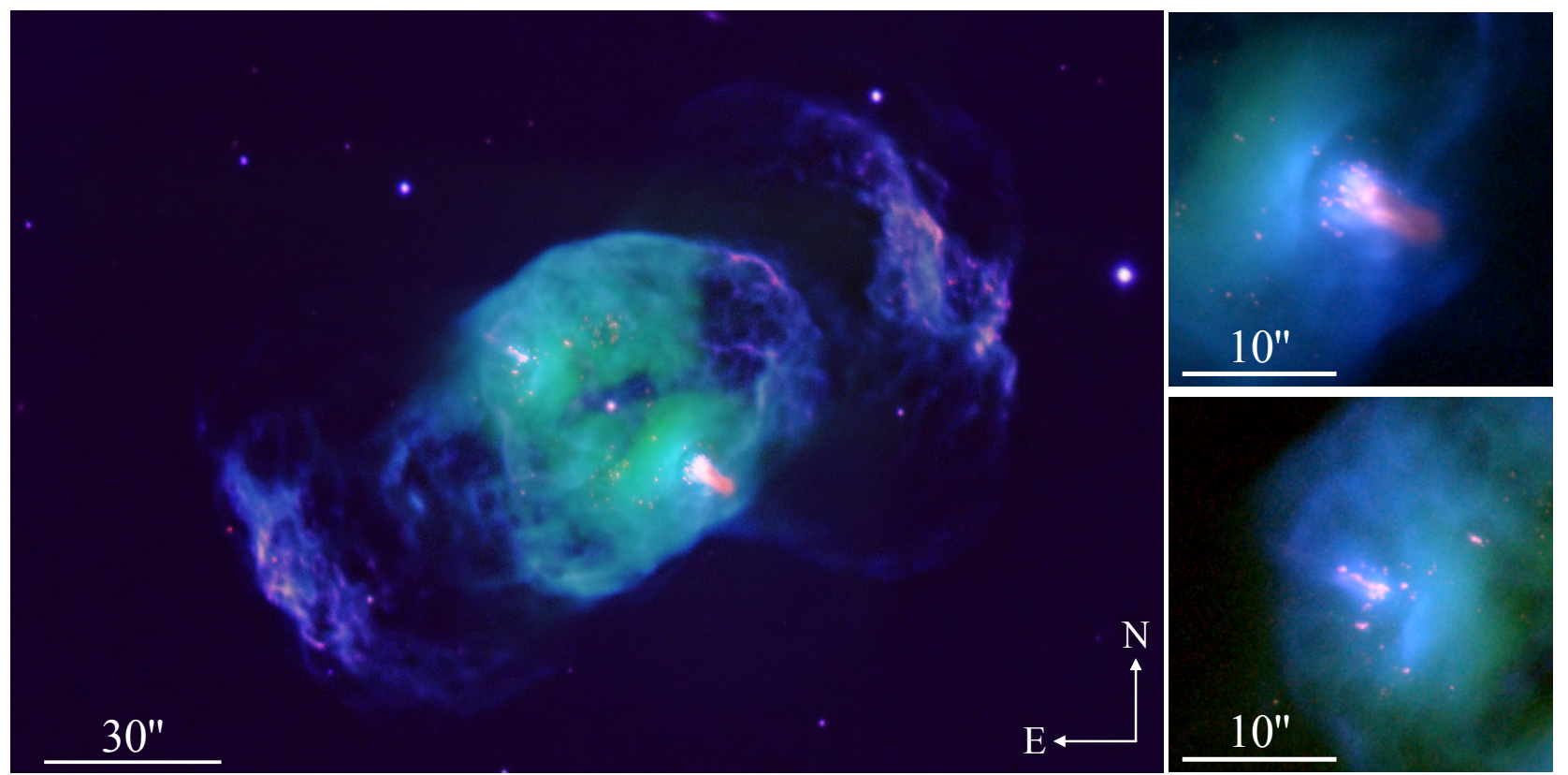

Figure 1. Colour-composite images of NGC 2371. The wide-field image (left panel) was produced by combining the CFHT and HST observations. The right panels were obtained by only taking the HST observations. In all panels red, green and blue correspond to [N II], H $\alpha$ and [O III], respectively.

PN has been identified only around a few [WR]-type CSPN (see Guerrero et al. 2018, and references therein). These are known as born-again $\mathrm{PNe}$ and it is accepted that the CSPN experienced a very late thermal pulse (VLTP) ejecting hydrogen-depleted and carbonrich processed material (e.g., Herwig et al. 1999; Miller Bertolami \& Althaus 2006). Although this evolutionary path has been suggested to explain the formation of hydrogen-poor [WR] stars, it might not apply to all PNe harboring [WR] stars (see Górny \& Tylenda 2000).

Statistical studies of PNe around [WR] CSPNe have shed light into the relation between the nebular properties and the evolutionary sequence of the progenitor stars. For example, the decrease in electron density $\left(n_{\mathrm{e}}\right)$ with decreasing spectral subtype has been suggested to be a signature of an evolutionary sequence from the late to early [WC]-type (Acker et al. 1996; Górny \& Tylenda 2000). Furthermore, the N/O abundance ratio vs. [WC] class presented by Peña et al. (2001) confirmed the claim that stars with different masses evolve through the same [WC] state (e.g., Peña et al. 1998; De Marco \& Crowther 1999).

Comparisons between $\mathrm{PNe}$ harboring non-emission line CSPNe with those hosting [WR] CSPNe have unveiled subtle differences. PNe with [WR] CSPNe are generally more centrally condensed, expand faster, and exhibit a higher degree of turbulence than PNe with non-emission line CSPNe (e.g., Gesicki et al. 2006; Medina et al. 2006; Jacob et al. 2013, and references therein). They also seem to have higher $\mathrm{N}$ and $\mathrm{C}$ abundances, implying that they have evolved from stars with initial masses $\sim 4 \mathrm{M}_{\odot}$ (García-Rojas et al. 2013). This is consistent with their preferential location at low latitudes in the Galactic disk (Peña et al. 2013). Finally, it has been noted recently that PNe with [WC] CSPNe have a higher occurrence of fast collimated outflows than $\mathrm{PNe}$ with weak emission line CSPNe or non-emission line CSPNe (Rechy-García et al. 2017).

These statistical studies rely on averaged values of the nebular physical properties (electron density and temperature), abundances, and velocity fields that might hide important clues on the evolution of PNe with [WR]-type CSPNe. For example, dedicated studies of compact [WR] PNe have unveiled the effects of fast collimated outflows in their early shaping (Rechy-García et al. 2017, 2019). Detailed studies of individual PNe with [WR] CSPNe are clearly needed to understand this unique evolutionary path of low- and intermediate-mass stars. We have therefore started a series of detailed studies of PNe harboring [WR] CSPNe. In this first paper we present a study of NGC 2371 (a.k.a. PNG 189.1+19.8; Curtis 1918), which harbors the [WR] CSPN WD 0722+295.

NGC 2371 has been classified as a barrel-like PNe of Peimbert type II (see table 1 in Henry et al. 2018). Morphological studies performed with the Hubble Space Telescope (HST), Spitzer and ground-based telescopes have unveiled a variety of morphological features (see Fig. 1; Sabbadin et al. 1982; Ramos-Larios \& Phillips 2012): two-bipolar lobes extending $\gtrsim 1$ arcmin from the CSPN towards the NW and SE directions with position angle $\mathrm{PA} \approx-60^{\circ}$, a main central cavity with an ellipsoidal shape with an extension of $28^{\prime \prime} \times 38^{\prime \prime}$ that exhibits a couple of blowout features aligned with the outer lobes, and a collection of dense knots mainly detected in $\left[\mathrm{N}_{\mathrm{II}}\right]$ and $\left[\mathrm{S}_{\mathrm{II}}\right]$ narrow-band filter images (see next section). The two brightest knots are aligned in the NE to SW direction with a $\mathrm{PA} \approx 60^{\circ}$, but the line that connects them is not perfectly aligned with the CSPN. Ramos-Larios \& Phillips (2012) presented a detailed discussion on the properties of the dense NE and SW knots. Their HST images show that these are actually conglomerates of clumps (see also Fig. 1). A secondary pair of smaller size clumps is observed at a $\mathrm{PA}=5^{\circ}$. It is worth noting that the low-ionisation clumps do not have a uniform distribution around the CSPN nor are they aligned with the bipolar lobes.

Optical (Torres-Peimbert \& Peimbert 1977; Aller \& Czyzak 1979) and International Ultraviolet Explorer (IUE) UV (Pottasch et al. 1981) observations of NGC 2371 have been used to estimate an averaged electron temperatures $\left(T_{\mathrm{e}}\right)$ in the range 9,000-23,000 K, depending on the line ratio used or the adopted electron density $\left(n_{\mathrm{e}}\right)$, which is found to be $800-2,500 \mathrm{~cm}^{-3}$ (e.g., Peña et al. 2001). Kaler et al. (1993) presented the analysis of the optical spectrum in the 
blue region ( 3700-5000 $\AA$ ) and showed it to be dominated by the O vi $\lambda \lambda 3811,3834$ doublet with contributions from the He II $\lambda 4686$, $\mathrm{C}_{\text {III }} \lambda 4650$ and $\mathrm{C}_{\text {IV }} \lambda 4658$. These authors estimated an effective temperature $\left(T_{\text {eff }}\right)$ for WD $0722+295$ of about $T_{\text {eff }}=[1-1.2] \times 10^{5} \mathrm{~K}$, consistent with the analysis of $I U E$ observations (Pottasch et al. 1981). Since its early classification as a [WR] star of the oxygen sequence (Smith \& Aller 1969), its spectral type has swung from early [WC3] (Heap 1982) to [WO1] (Acker \& Neiner 2003).

In this work, we intend to unveil the physical structure of the different components of NGC 2371 and to produce an accurate model of its central star. We have obtained Isaac Newton Telescope (INT) spectroscopic observations to study the physical properties (density and temperature) and abundances to describe the ionisation structure of the different morphological components of NGC 2371. In conjunction with archival IUE observations, the INT spectra are also used to perform a detailed characterisation of its CSPN. The paper is organized as follows. In Section 2 we describe the observations used in the present work. Section 3 presents the analysis and atmospheric model of WD $0722+295$. In Section 4 we present our spectral analysis of NGC 2371 as well as the estimates of the physical structure. In Section 5 we discuss our results and, finally, a summary is presented in Section 6.

\section{OBSERVATIONS}

Narrow-band images of NGC 2371 were downloaded from the archives of the Canada-France-Hawaii Telescope $(\mathrm{CFHT})^{1}$ and $H S T$ Legacy Archive ${ }^{2}$. The CFHT images were acquired with the multi-object spectrograph (MOS) camera. These images were obtained through the $\mathrm{H} \alpha$, [O III] and [N $\mathrm{II}]$ filters with total exposure times of $1200 \mathrm{~s}$ on each filter on December 18, 2002 (Prop. ID.: 02BC17; PI: S. Kwok). The HST images were obtained through the F502N, F656N, and F658N filters (hereafter [O III], $\mathrm{H} \alpha$ and [N II]) on 2007 November 15 with exposure times on each filter of $1600 \mathrm{~s}$ (Prop.ID. 11093, PI: K. Noll). A colour-composite image obtained by combining all these optical images is shown in Figure 1.

Long-slit spectroscopic observations were obtained at the INT of the Observatorio de El Roque de los Muchachos (La Palma, Spain) using the Intermediate Dispersion Spectrograph (IDS) in low- and high-dispersion spectral modes (PI: M. A. Guerrero). The high-resolution spectra were obtained on 2018 October 24 using the EEV10 CCD camera with the R1200U grating. This configuration has a dispersion of $0.48 \AA$ pixel $^{-1}$ over the $3200-4500 \AA$ wavelength range and a plate scale of 0 !' $^{\prime} 4$ pixel $^{-1}$. The low-resolution spectra were acquired on 2018 November 15 using the RED+2 CCD camera with the R400V grating. This configuration has a dispersion of $1.55 \AA$ pixel $^{-1}$ over the $3500-8000 \AA$ wavelength range and a similar

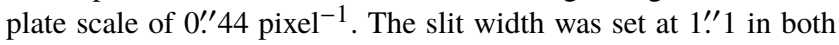
observing runs. Two slits at $\mathrm{PA}=65^{\circ}$ (Slit A) and $\mathrm{PA}=128^{\circ}$ (Slit B) were used with both spectral configurations. Slit B is centered on WD 0722+295, whereas Slit A probes the low-ionisation knots and therefore it does not goes exactly across the CSPN. The positions of these slits are overlaid on the colour-composite image of NGC 2371 in Figure 2. The total integration time on each position and spectral configuration was $900 \mathrm{~s}$. All spectra were analysed following IRAF standard routines. The wavelength calibration was performed using $\mathrm{CuAr}+\mathrm{CuNe}$ lamps.

\footnotetext{
${ }^{1}$ http://www.cadc-ccda.hia-iha.nrc-cnrc.gc.ca/en/

2 https://hla.stsci.edu/
}

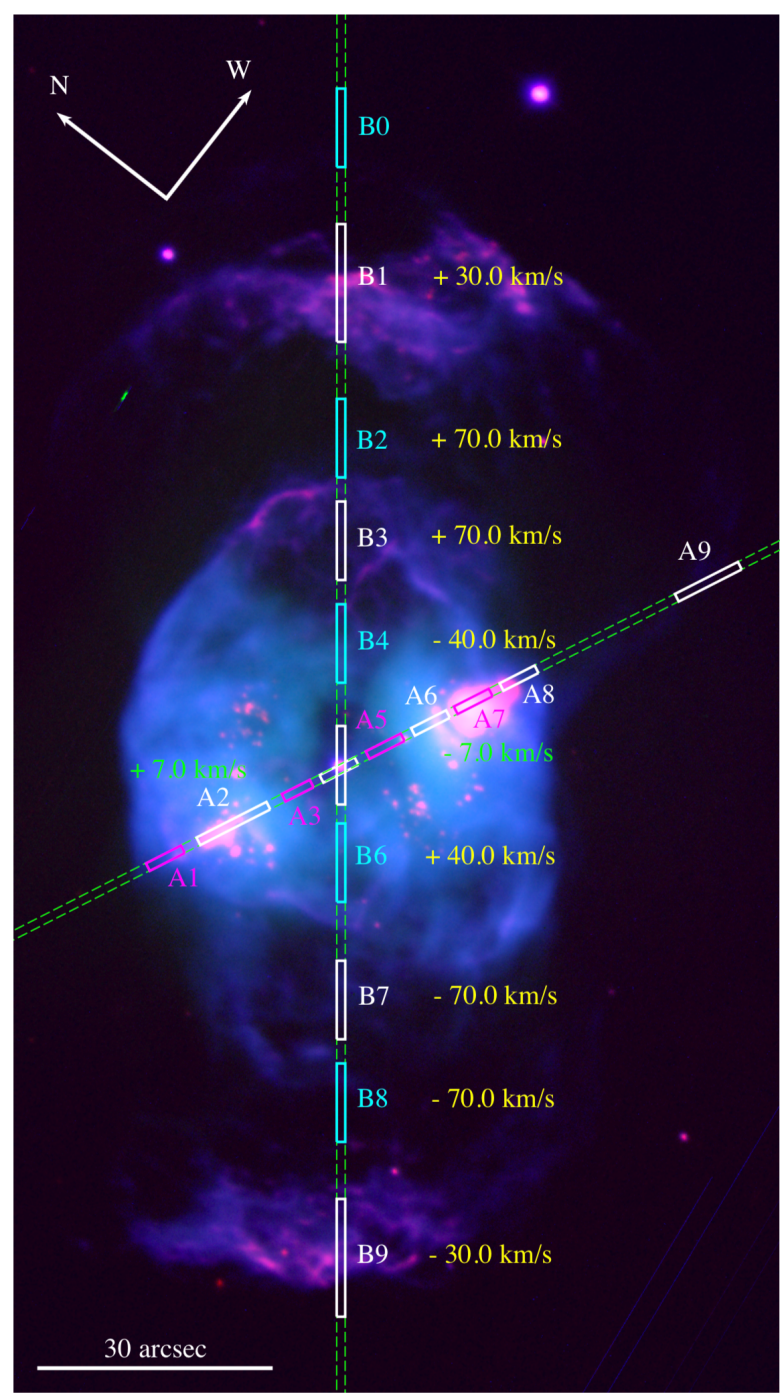

Figure 2. CFHT colour-composite ([O $\left.\mathrm{O}_{\mathrm{III}}\right]$ - blue, $\mathrm{H} \alpha$ - green, $\left[\mathrm{N}_{\mathrm{II}}\right]$ - red) image of NGC 2371. The (green) dashed-line regions represent the slits positions obtained from our INT IDS observations. The PA for the slits are $65^{\circ}$ (Slit A - minor axis) and $128^{\circ}$ (Slit B - major axis). Different extraction regions are labeled and shown with different colours. The green and yellow velocity measurements correspond to the A2, A7 and B1-B9 extraction regions. See text for details.

In order to study the ionisation structure of NGC 2371, we have extracted spectra from different regions defined on each slit using the IRAF task apall. Those extracted from Slit A were used to study the central main cavity of NGC 2371 and the dense knots located $\gtrsim 16^{\prime \prime}$ from the CSPN, and those from Slit B to study the outermost lobes and different regions of the central cavity along the NW-SE direction. All regions, labeled from A1-A9 and B0-B9, are shown in Figure 2 and their sizes are indicated in Tables 3 and 4 , respectively. We first extracted the 1-D spectrum of the CSPN (regions A4 and B5) tracing the stellar continuum along the 2-D spectrum. This information was then used as a reference to trace the nebular spectra (regions A1-A3, A5-A9, B0-B4, and B6-B9) along the 2-D spectra, as these regions does not show continuum emission. Since Slit A misses some stellar flux, the spectrum extracted for region A4 has not been used for stellar modelling. Finally, we note that all extracted spectra were corrected for extinction by using 


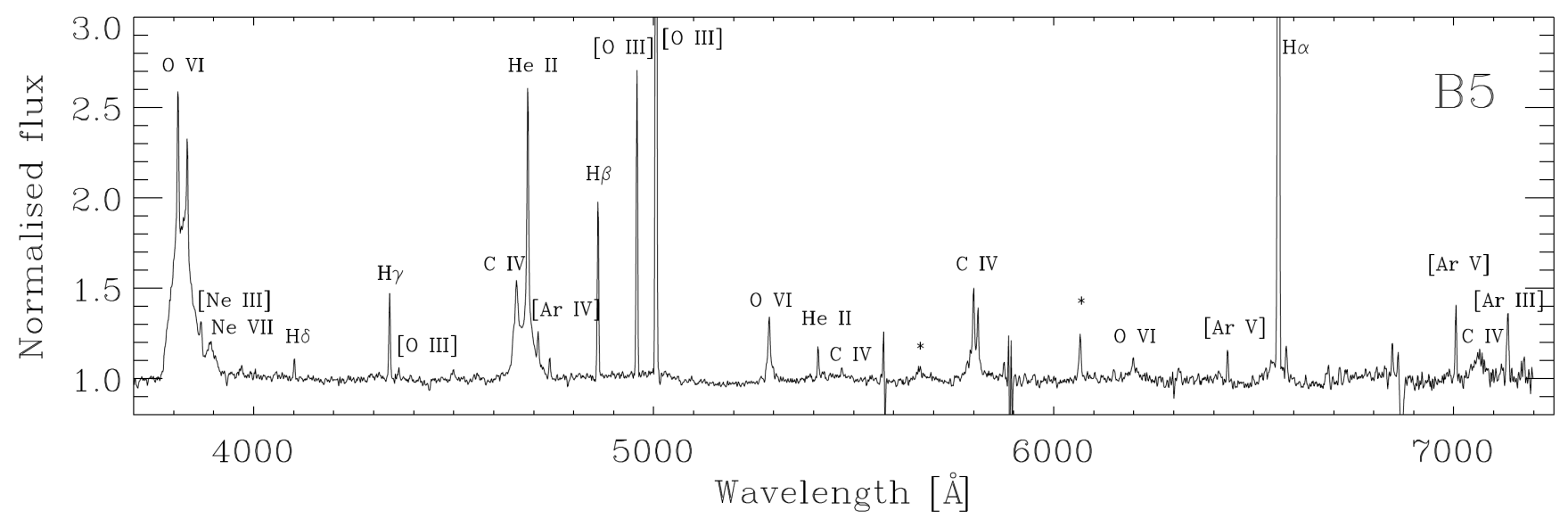

Figure 3. INT IDS low-resolution, normalised spectrum of the CSPN of NGC 2371 obtained from slit B. The most prominent WR features and nebular lines are marked.

the $c(\mathrm{H} \beta)$ value estimated from the Balmer decrement method. We assume intrinsic Balmer decrement ratio corresponding to a case B photoionised nebula of $T_{\mathrm{e}}=10,000 \mathrm{~K}$ and $n_{\mathrm{e}}=100 \mathrm{~cm}^{-3}$ (see Osterbrock \& Ferland 2006) and the reddening curve of Cardelli et al. (1989).

\section{WD 0722+295 - THE CSPN OF NGC 2371}

The optical spectrum of WD $0722+295$ obtained from our INT IDS observations is presented in Figure 3. The stellar spectrum exhibits the classic WR blue and red bumps (BB and RB) at $\sim 4686 \AA$ and $\sim 5806 \AA$, respectively, as well as a broad O vi feature at $\sim 3820 \AA$ (see also figure 1 in Kaler et al. 1993). Other emission lines related to WR features such as those reported in Acker \& Neiner (2003) namely, O vi $5290 \AA$, C Iv $5470 \AA$ and $7060 \AA$, and He II $5412 \AA$ are also present in the optical spectrum. Furthermore, we also detect the O vi $6200 \AA$ as a broad emission line. All identified WR features as well as some contributing nebular lines are marked in Figure 3. We note the presence of narrow emission lines at $\approx 5665 \AA$ and $\approx 6066 \AA$ marked with an asterisk in Figure 3 and Figure 4. There has been controversy in the identification of these lines, which can be assigned either to the $\mathrm{O}$ VII $\lambda 5666$ and $\mathrm{O}$ VIII $\lambda 6068$ lines or to the Ne VII $\lambda 5666$ and Ne VIII $\lambda 6068$ lines. This is discussed in Section 5.

To provide an appropriate description of the spectrum and spectral classification of WD $0722+295$, the contribution from nebular lines blended with the broad WR features needs to be accounted for. This was done by applying the analysis described by GómezGonzález et al. (2020). This method consists on fitting the broad WR features with multi-Gaussian components using a tailor-made code that uses the IDL routine LMFIT ${ }^{3}$. As a result, fluxes, central wavelength, FWHM and equivalent widths (EW) from the WR spectral features as well as contributing nebular lines have been estimated. All these parameters are listed in Table 1. We note that those lines listed as WR features have FWHM larger than those of the nebular narrow lines, that is, FWHM $>4.5 \AA$.

3 The LMFIT function (lmfit.pro) performs a non-linear least squares fit to a function with an arbitrary number of parameters. It uses the LevenbergMarquardt algorithm, incorporated in the routine mrqmin from Press et al. (1992).
Table 1. Parameters for the emission lines considered for the multi-Gaussian fitting of the WR features of the central star in NGC 2371.

\begin{tabular}{|c|c|c|c|c|c|c|c|c|}
\hline $\begin{array}{l}\text { ID } \\
\text { (1) }\end{array}$ & (2) & $\begin{array}{l}\text { Ion } \\
\text { (3) }\end{array}$ & $\begin{array}{l}\lambda_{0} \\
(4)\end{array}$ & $\begin{array}{l}\mathrm{F} \\
(5)\end{array}$ & $\begin{array}{c}\text { FWHM } \\
(6)\end{array}$ & $\begin{array}{c}\text { EW } \\
(7)\end{array}$ & $\begin{array}{l}\lambda_{c} \\
(8)\end{array}$ & $\begin{array}{c}F / F_{\mathrm{RB}} \\
\quad(9)\end{array}$ \\
\hline \multicolumn{9}{|l|}{ BB } \\
\hline 1 & WR & He II & 4686 & 11.4 & 28.2 & 11.2 & 4686.0 & 712.5 \\
\hline 2 & WR & $\mathrm{C}_{\text {IV }}$ & 4658 & 7.3 & 15.9 & 7.0 & 4656.9 & 456.3 \\
\hline 3 & $\mathrm{Neb}$ & He II & 4686 & 5.8 & 4.5 & 5.7 & 4685.2 & $\ldots$ \\
\hline 4 & $\mathrm{Neb}$ & Ar IV & 4711 & 0.9 & 4.5 & 0.9 & 4711.5 & $\ldots$ \\
\hline \multicolumn{9}{|l|}{$\mathrm{RB}$} \\
\hline 5 & WR & $\mathrm{C}_{\mathrm{IV}}$ & 5801 & 1.0 & 7.1 & 3.1 & 5799.7 & $\cdots$ \\
\hline 6 & WR & $\mathrm{C}_{\text {IV }}$ & 5812 & 0.6 & 5.4 & 1.9 & 5810.5 & $\ldots$ \\
\hline \multicolumn{9}{|l|}{ OB } \\
\hline 7 & WR & O VI & 3820 & 117.6 & 57.5 & 53.2 & 3818.7 & 7350.0 \\
\hline 8 & $\mathrm{Neb}$ & $\mathrm{OVII}_{\mathrm{V}}$ & 11 & 8.0 & 4.2 & 3.6 & 3810.9 & $\ldots$ \\
\hline 9 & Neb & O vI & 3834 & 5.7 & 4.1 & 2.6 & 3834.0 & .. \\
\hline \multicolumn{9}{|c|}{ other } \\
\hline 10 & WR & O VI & 5290 & 1.7 & 9.6 & 3.2 & 5288.7 & 106.3 \\
\hline 11 & $\mathrm{Neb}$ & He II & 5412 & 0.3 & 3.6 & 0.7 & 5411.1 & $\ldots$ \\
\hline 12 & WR & $\mathrm{C}_{\text {IV }}$ & 5470 & 0.1 & 4.6 & 0.3 & 5470.4 & 6.3 \\
\hline 13 & WR & $\mathrm{Ne}$ VII ${ }^{\dagger}$ & 5666 & 0.3 & 8.2 & 0.8 & 5664.6 & 18.8 \\
\hline 14 & WR & $\mathrm{Ne}$ VIII ${ }^{\dagger}$ & 6068 & 0.4 & 6.4 & 1.6 & 6066.0 & 25.0 \\
\hline 15 & WR & OVI & 6200 & 0.4 & 18.0 & 1.4 & 6200.0 & 25.0 \\
\hline 16 & WR & $\mathrm{C}_{\text {IV }}$ & 7060 & 3.1 & 30.3 & 3.5 & 7062.5 & 193.8 \\
\hline
\end{tabular}

(1) Identification number of the Gaussian components in the WR features; (2) Nature of the contributing emission line: WR (broad) or nebular (narrow); (3) Ion responsible for the line; (4) Rest wavelength in $\AA$; (5) Flux in units of $10^{-14} \mathrm{erg} \mathrm{cm}^{-2} \mathrm{~s}^{-1}$; (6) Full Width at Half Maximum (FWHM) $[\AA ̊]$; (7) Equivalent Width (EW) [Å]; (8) Observed center of the line; (8) Line fluxes normalised to the RB. The ratio has been computed adopting a $F(\mathrm{RB})=100{ }^{\dagger}$ This identification is discussed in Section 5.

Figure 4 shows the fits to the different WR features of the WD $0722+295$. Two of these bumps are composed of several blended emission lines and need a careful modelling in order to separate their broad and narrow contributions. The O-bump feature is made of a broad O VI (FWHM of $57.5 \AA$ ), two O VI narrow lines $(\mathrm{FWHM}<4.5 \AA$ ) and several Ne lines at the red wing (see Table 1). It is clear that the broad O vi $3820 \AA$ has a stellar origin and, although the other two O vi lines have FWHM that suggest a nebular origin, some contribution from the star is expected according to our stellar atmosphere model (see Section 3.1 and Fig. 5). The 

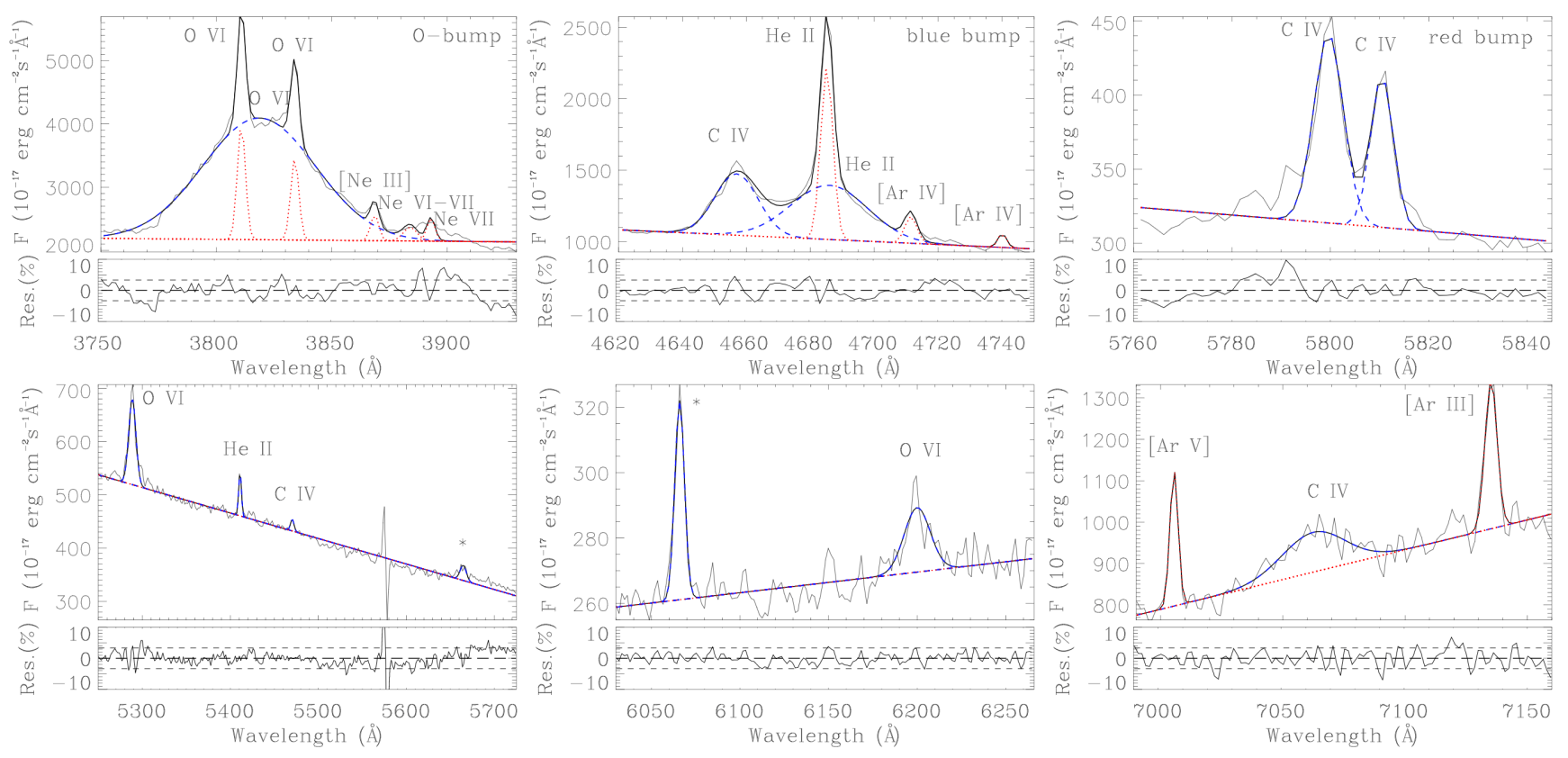

Figure 4. Multi-component Gaussian fits to the O-bump (top left), blue bump (top center) and red bump (top right) panels. The bottom panels show other WR features in the spectrum of WD $0722+295$. The (blue) dashed lines represents the fits to the broad (stellar) features, the (red) dotted-line the fits to the nebular emission and the (black) line the sum. Residuals are shown at the bottom of each panel.

Gaussian-fitting method shows that the O vi lines at $3811 \AA$ and $3834 \AA$ A contribute about $10 \%$ to the O-bump. Thus, this can be considered as a upper limit to the nebular contribution. Other narrow emission lines also contribute to the BB. For example, the He II, which is blended with WR features contributes $\simeq 30 \%$ of the total flux of the $\mathrm{BB}$ (He II $4686 \AA+\mathrm{C}$ iv $4658 \AA$; see Table 2). There is no contribution of nebular lines to the RB (C IV $\left.5801 \AA+C_{\text {IV }} 5812 \AA\right)$.

With reliable flux estimates for each WR feature, we can assess the spectral classification of WD $0722+295$. For this, we estimated the line ratios of oxygen, carbon and helium WR features relative to the RB (Column 9 of Table 1) and compared them to those defining the quantitative classification scheme of [WR] stars listed in table 2 of Acker \& Neiner (2003). According to this scheme, WD $0722+295$ fulfills the criteria for a [WO1]-type star, but we note that the FWHM of the RB is around half of that suggested for this spectral type. One could argue that using dereddened flux ratios is model dependent and less accurate than using EW ratios. For this, we also compared our results listed in Table 1 with the classification scheme proposed in Crowther et al. (1998) and found that the EW ratio of the $\mathrm{O}$ vi $3820 \AA$ WR feature over the $\mathrm{RB}$ is 73.5 , above the limit of $\sim 1.6$ suggested by these authors for a [WO1] spectral type.

\subsection{NLTE analysis of WD $0722+295$}

To obtain a more quantitative description of the properties of WD $0722+295$, we have analysed optical and UV spectra by means of the updated version of the Potsdam Wolf-Rayet (PoWR) ${ }^{4}$ NLTE stellar atmosphere code (Gräfener et al. 2002; Hamann \& Gräfener 2004). Details of the computing scheme can be found in Todt et al. (2015) and the most recent example of the capabilities of the PoWR

${ }^{4}$ http://www.astro.physik.uni-potsdam.de/ wrh/PoWR code performed by our group can be found in Toalá et al. (2019) for the case of the [WR]-type CSPN of NGC 40.

Available UV Far Ultraviolet Spectroscopic Explorer (FUSE) and IUE observations of WD 0722+295 were retrieved from the Mikulski Archive for Space Telescopes ${ }^{5}$. The UV observations were analysed in conjunction with the optical INT IDS spectra with the PoWR stellar atmosphere code. The FUSE observations correspond to Obs. ID. p1330301000 (PI: L. Bianchi) and were obtained on 2000 February 26 with the LWRS aperture for a total exposure time of 5259 s. The IUE data, taken on 1979 April 7, correspond to the Obs. ID. SWP04883 and LRW04210 (Program ID SP127; PI: S. R. Pottasch) with exposure times of $1560 \mathrm{~s}$ and $2700 \mathrm{~s}$, respectively. The spectra imply a reddening of $E(B-V)=0.08 \mathrm{mag}$ using the Cardelli extinction law which is consistent with the value estimated by Herald \& Bianchi (2004).

The parameters of our best-fit model are listed in Table 2 . We note that the stellar mass was adopted to be $M_{\star}=0.6 \mathrm{M}_{\odot}$, a typical value for CSPNe (e.g., Miller Bertolami 2016, and references therein). The resultant temperature, defined at $\tau_{\text {Ross }}=20$, which is constrained by the relative strength of the emission lines, is $T_{\text {eff }}=130 \mathrm{kK}$. A luminosity of $L_{\star}=2820 \mathrm{~L}_{\odot}$ is estimated by using a distance $d=1.75 \mathrm{kpc}$ (Bailer-Jones et al. 2018) and the resultant mass-loss rate is $\dot{M}=1.78 \times 10^{-8} \mathrm{M}_{\odot} \mathrm{yr}^{-1}$. We note that the estimated value for the mass-loss rate is somewhat smaller than those listed for other [WR] stars in Todt et al. (2013b), who used a canonical luminosity of $L=5000 L_{\odot}$ to calculate the mass-loss rates from the $R_{\mathrm{t}}$ values obtained from the analysis. Taking the lower luminosity of NGC 2371 into account, our value of $\dot{M}$ is comparable with the one derived for Hen 2-55. Furthermore, our $R_{\mathrm{t}}=20 R_{\odot}$ is fully compatible with the value of $R_{\mathrm{t}}=15_{-5}^{+10} R_{\odot}$ by Herald \& Bianchi (2004). They used the same luminosity as in our analysis 

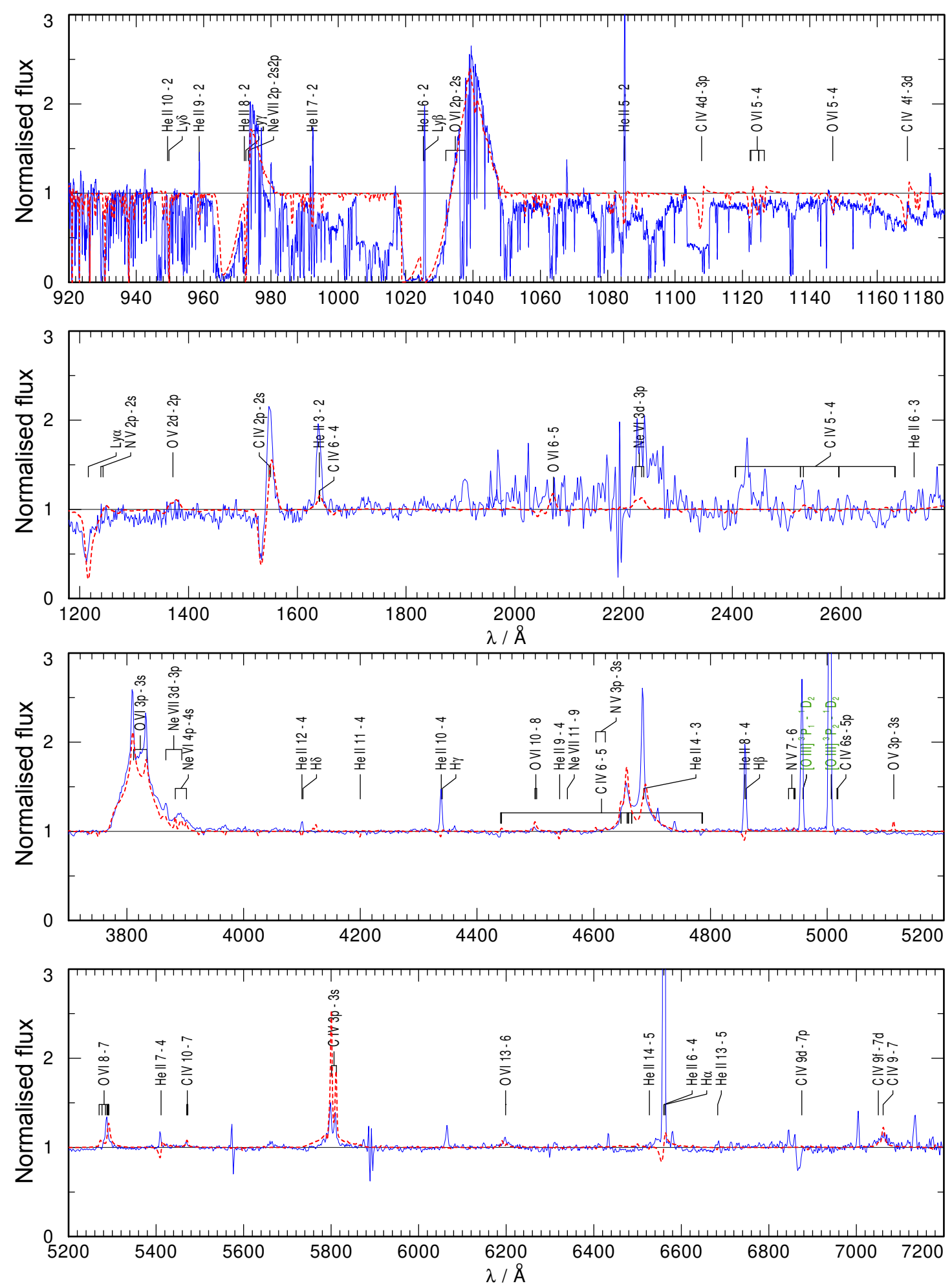

Figure 5. Comparison between our PoWR model (red dashed line) with optical and UV observations (blue solid line).

(based on the distance of $d=1.5 \mathrm{kpc}$ ), but assumed a smooth wind with $D=1$, where we adopted a density contrast of $D=10$ for a clumped wind. Therefore, their mass-loss rate is about a factor $\sqrt{10}$ larger than ours.

Our best-fit model to the P Cygni profiles in the UV spectra resulted in a stellar wind velocity $v_{\infty}=3700 \mathrm{~km} \mathrm{~s}^{-1}$ with a micro- turbulence of less than $3 \%$. The micro-clumping parameter $D$, which is defined as the density contrast between a smooth wind and a clumpy wind, is estimated to be 10 . Figure 5 shows a comparison between the PoWR model of WD 0722+295 and the optical and UV spectra, and Figure 6 presents the synthetic spectral energy distribution (SED) from the far-UV to the IR in comparison with 


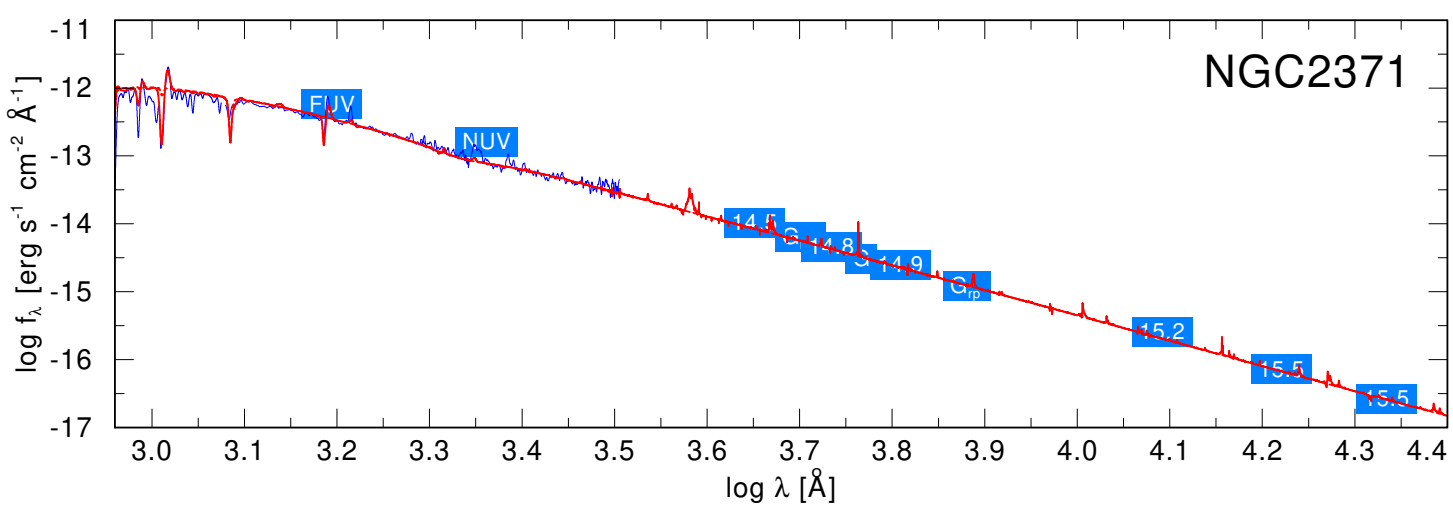

Figure 6. Comparison between our PoWR model (red line) with observed spectra in the optical and UV and IR photometry (blue).
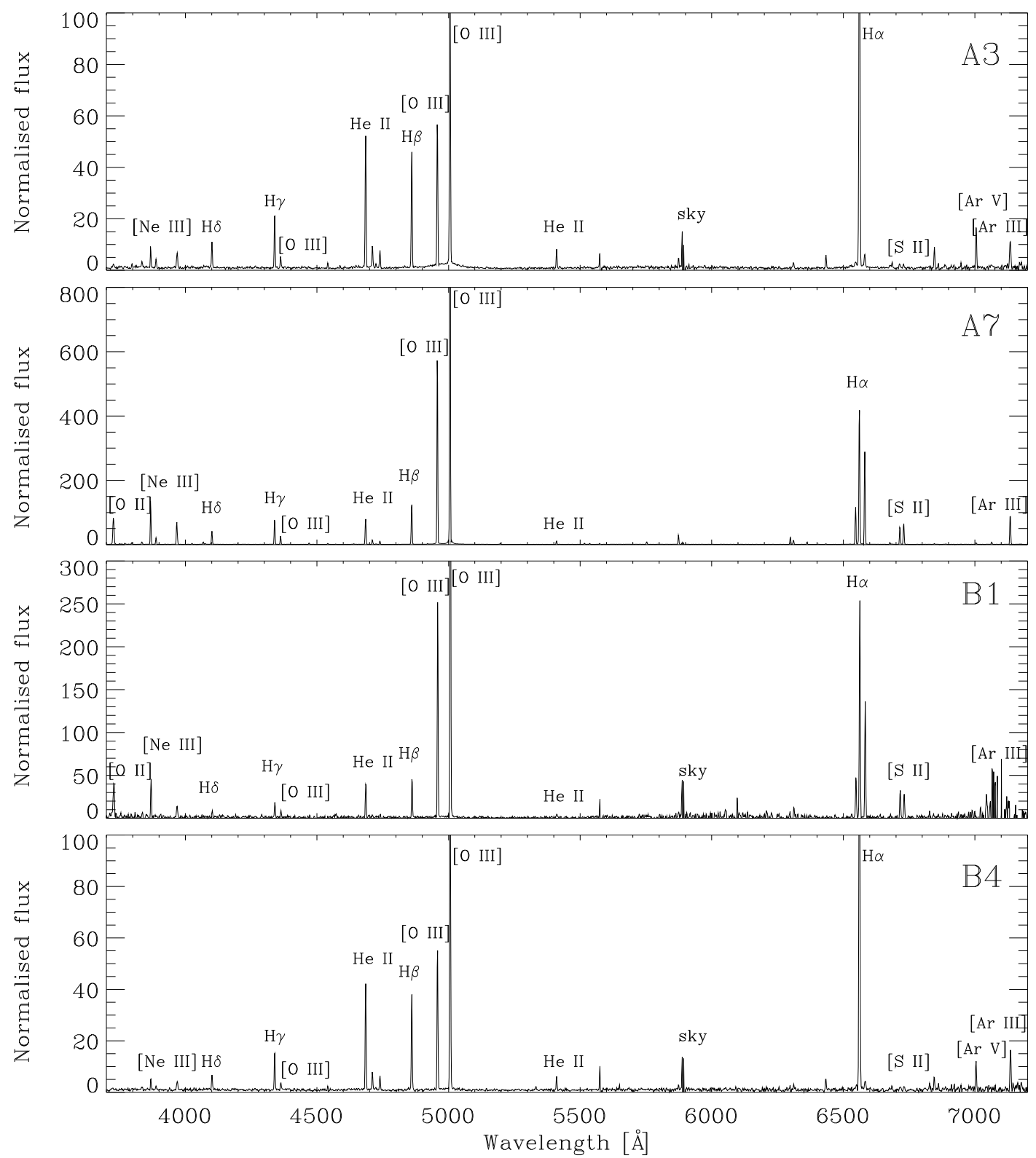

Figure 7. Examples of INT IDS spectra of NGC 2371. The panels show the spectra extracted from regions A3, A7, B1 and B4. The most prominent lines are labeled. See Tables 3 and 4 for details on the fluxes. 
Table 2. Parameters of WD $0722+295$ obtained with PoWR.

\begin{tabular}{lcl}
\hline Parameter & Value & Comment \\
\hline$T_{\text {eff }}[\mathrm{kK}]$ & 130 & Defined at $\tau_{\text {Ross }}=20$ \\
$d[\mathrm{kpc}]$ & 1.75 & Bailer-Jones et al. $(2018)$ \\
$\log \left(L_{\star} / L_{\odot}\right)$ & 3.45 & \\
$R_{\star}\left[R_{\odot}\right]$ & 0.105 & \\
$R_{\mathrm{t}}\left[R_{\odot}\right]$ & 20 & \\
$D$ & 10 & Density contrast \\
$\log \left(\dot{M} / \mathrm{M}_{\odot} \mathrm{yr}^{-1}\right)$ & -7.75 & for $D=10$ \\
$v_{\infty}\left[\mathrm{km} \mathrm{s}^{-1}\right]$ & 3700 & \\
$M_{\star}\left[\mathrm{M}_{\odot}\right]$ & 0.6 & Stellar mass adopted \\
\hline $\mathrm{Chemical} \mathrm{abundances}(\mathrm{mass}$ fraction $)$ \\
\hline $\mathrm{He}$ & 0.71 & \\
$\mathrm{C}$ & 0.20 & \\
$\mathrm{~N}$ & 0.001 & \\
$\mathrm{O}$ & 0.06 & \\
$\mathrm{Ne}$ & 0.03 & \\
$\mathrm{Fe}$ & $1.4 \times 10^{-3}$ & \\
\hline
\end{tabular}

UV and optical spectra and IR photometry obtained from public archives.

The abundances for WD $0722+295$ derived from our best-fit model are also listed in Table 2. Whereas the stellar parameters agree with those reported by Herald \& Bianchi (2004, see their table 6), our abundance determinations are at variance, with our He abundance $30 \%$ larger and our N/O ratio half their value. The differences can be attributed to the use of different stellar atmosphere codes, as Herald \& Bianchi (2004) use a relatively old version of the CMFGEN code (Hillier \& Miller 1998, 1999), but we note that our abundance determination is improved by the simultaneous modelling of high-quality optical spectra and UV data.

\section{PHYSICAL CONDITIONS, EXCITATION AND CHEMICAL ABUNDANCES OF NGC 2371}

Examples of INT IDS spectra extracted from Slits A and B are shown in Figure 7. The complete list of lines detected in the different extraction regions and their dereddened intensities relative to an arbitrary value 100 for $\mathrm{H} \beta$ are presented in Tables 3 and 4. The spectra extracted from Slit B, particularly those from regions B2, B3, B7, and B8, show fewer emission lines than those extracted from Slit A, in agreement with the lower surface brightness of the major axis probed by Slit B (see Fig. 1). Intensity uncertainties at $1-\sigma$ are provided in these tables, together with the values derived for $c(\mathrm{H} \beta)$ and the corresponding fluxes of the $\mathrm{H} \beta$ line for each extraction region.

Different line intensity ratios have been used to determine the physical conditions of the different regions in NGC 2371 using the IRAF task temden (Tody 1993). $T_{\mathrm{e}}$ was estimated using the [O III] emission lines for most regions and the $\left[\mathrm{N}_{\mathrm{II}}\right]$ emission lines whenever possible, i.e., in regions $\mathrm{A} 2, \mathrm{~A} 7$, and $\mathrm{A} 8$. $n_{\mathrm{e}}$ was derived using the $\left[\mathrm{S}_{\mathrm{II}}\right] \lambda \lambda 6717,6731,\left[\mathrm{Cl}_{\text {III }}\right] \lambda \lambda 5517,5537$, and [Ar IV] $\lambda \lambda 4711,4740$ doublets whenever available. The values of $T_{\mathrm{e}}$ and $n_{\mathrm{e}}$, together with their 1- $\sigma$ uncertainties are listed in the bottom rows of Tables 3 and 4 .

The values of $T_{\mathrm{e}}\left(\left[\mathrm{O}_{\mathrm{III}}\right]\right)$ listed in Tables 3 and 4 reveal notable temperature gradients in the central cavity of NGC 2371. The innermost regions $\mathrm{A} 2, \mathrm{~A} 3, \mathrm{~A} 5, \mathrm{~A} 5, \mathrm{~B} 4$, and $\mathrm{B} 6$ immediately around the CSPN show consistently high values of $T_{\mathrm{e}}\left(\left[\mathrm{O}_{\mathrm{III}}\right]\right)$ in the range
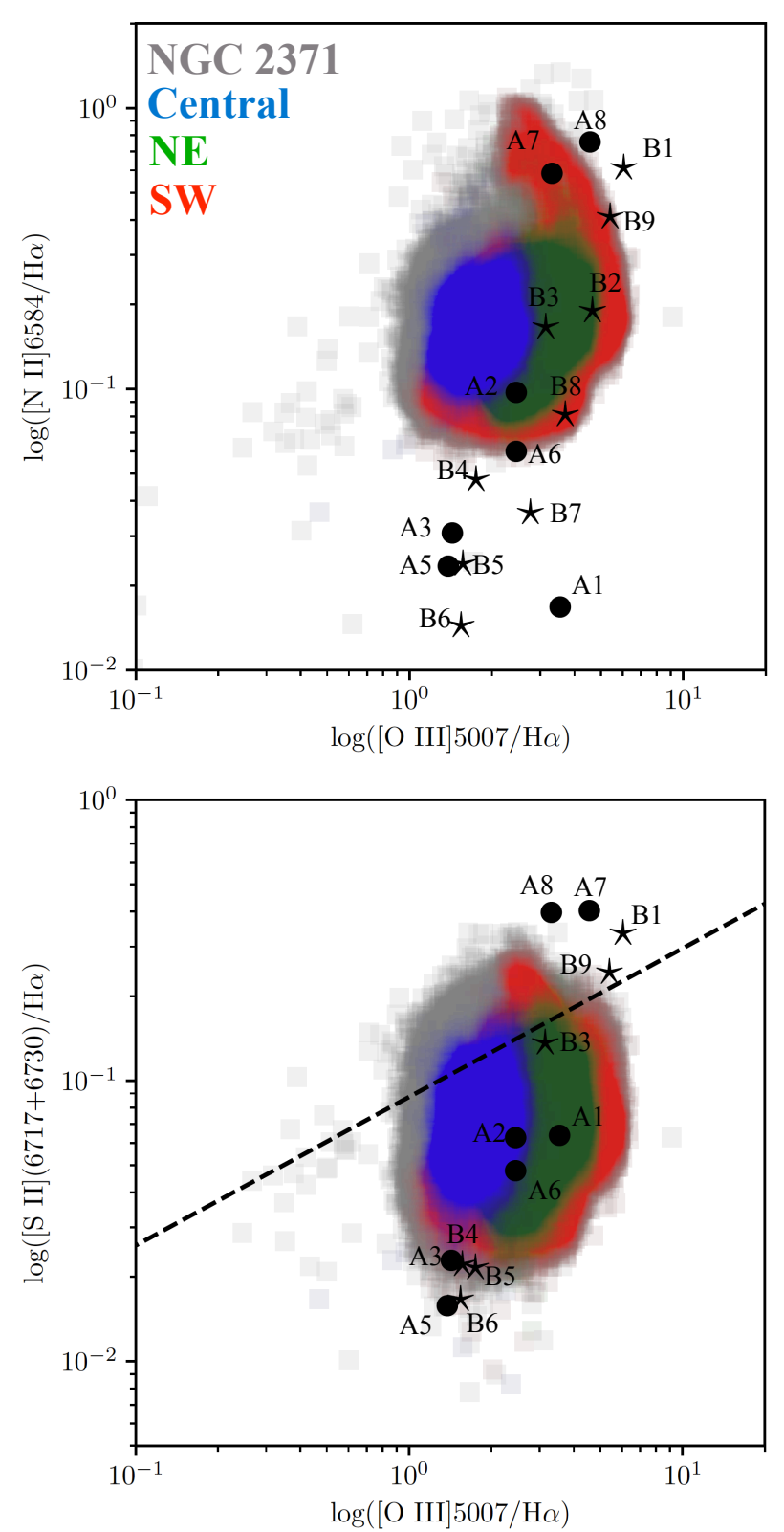

Figure 8. Line intensity ratios derived for spectra extracted from the regions in Slit A (bullets) and Slit B (stars), and from different regions of HST flux-calibrated ratio maps as described by the coloured points. Different colours represent line ratios extracted from the regions defined in Figure 9 left panel. The dashed line in the bottom panel marks the theoretical limit $\log \left(\left[\mathrm{O}_{\mathrm{II}}\right] / \mathrm{H} \alpha\right)=1.89 \times \log \left(\left[\mathrm{S}_{\mathrm{II}}\right] / \mathrm{H} \alpha\right)+2.46$ (see Section 4 for details) between photoionization (below the line) and shock-excitation (above the line).

$18,000-19,000 \mathrm{~K}$, that decrease to $\approx 15,000-16,000 \mathrm{~K}$ in the outermost regions $\mathrm{A} 1$ and $\mathrm{A} 8$, and have the notably lowest value of $\approx 13,800 \mathrm{~K}$ in the region $\mathrm{A} 7$, which probes the SW knot.

Such high values of $T_{\mathrm{e}}$ in the close vicinity of the CSPN of NGC 2371 arise from its $130 \mathrm{kK}$ high $T_{\text {eff }}$ (see Section 3.1). Interestingly, $T_{\mathrm{e}}([\mathrm{N} \mathrm{II}])$ is only computed on regions that probe the $\mathrm{NE}$ and $\mathrm{SW}$ knots. The much lower values of $T_{\mathrm{e}}\left(\left[\mathrm{N}_{\mathrm{II}}\right]\right)$ in these regions with 
Table 3. Reddeding-corrected line intensities for selected regions of Slit A of NGC 2371

\begin{tabular}{|c|c|c|c|c|c|c|c|c|c|}
\hline$\lambda_{0}$ & line & $\begin{array}{l}\text { A1 } \\
\left(5^{\prime \prime}\right)\end{array}$ & $\begin{array}{c}\text { A2 } \\
\left(10^{\prime \prime}\right)\end{array}$ & $\begin{array}{c}\text { A3 } \\
\left(4^{\prime \prime}\right)\end{array}$ & $\begin{array}{l}\text { A5 } \\
\left(5^{\prime \prime}\right)\end{array}$ & $\begin{array}{c}\text { A6 } \\
\left(5^{\prime \prime}\right)\end{array}$ & $\begin{array}{l}\text { A7 } \\
\left(5^{\prime \prime}\right)\end{array}$ & $\begin{array}{c}\text { A8 } \\
\left(5^{\prime \prime}\right)\end{array}$ & $\begin{array}{c}\text { A9 } \\
\left(10^{\prime \prime}\right)\end{array}$ \\
\hline 3726.2 & {$\left[\mathrm{O}_{\mathrm{II}}\right]$} & $33.2 \pm 5.4$ & $35.3 \pm 1.8$ & $11.6 \pm 2.4$ & $16.5 \pm 3.0$ & $24.1 \pm 1.7$ & $201.2 \pm 4.9$ & $141.7 \pm 8.1$ & $\ldots$ \\
\hline 3797.9 & $\mathrm{H} \theta$ & $8.4 \pm 3.2$ & $6.5 \pm 0.7$ & $6.9 \pm 1.7$ & $7.2 \pm 1.5$ & $5.7 \pm 0.6$ & $8.6 \pm 0.8$ & $\ldots$ & $\ldots$ \\
\hline 3835.4 & $\mathrm{H} \eta$ & $13.5 \pm 3.9$ & $9.6 \pm 0.8$ & $12.3 \pm 2.1$ & $12.2 \pm 1.8$ & $9.9 \pm 0.6$ & $9.3 \pm 0.7$ & & $\ldots$ \\
\hline 3868.8 & [Ne III] & $99.4 \pm 7.0$ & $81.3 \pm 1.9$ & $32.6 \pm 2.2$ & $33.5 \pm 2.2$ & $74.7 \pm 1.2$ & $157.4 \pm 2.3$ & $97.5 \pm 6.1$ & $\ldots$ \\
\hline 3889.1 & $\mathrm{H} \zeta$ & $16.5 \pm 3.5$ & $16.0 \pm 0.9$ & $12.6 \pm 1.7$ & $14.9 \pm 1.5$ & $16.2 \pm 0.6$ & $21.9 \pm 0.8$ & $15.6 \pm 2.7$ & $\ldots$ \\
\hline 3967.5 & {$[\mathrm{Ne} \mathrm{III}]$} & $49.8 \pm 5.0$ & $44.3 \pm 1.4$ & $24.4 \pm 2.2$ & $29.5 \pm 2.0$ & $43.7 \pm 1.0$ & $72.1 \pm 1.4$ & $46.1 \pm 3.7$ & $\ldots$ \\
\hline 3970.0 & $\mathrm{H} \epsilon$ & $\ldots$ & $\ldots$ & $\ldots$ & $\ldots$ & $\ldots$ & $\ldots$ & $\ldots$ & $\ldots$ \\
\hline 4026.2 & $\mathrm{He}_{\mathrm{I}}$ & $4.6 \pm 2.6$ & $2.0 \pm 0.4$ & $1.2 \pm 0.8$ & $2.7 \pm 1.1$ & $2.2 \pm 0.4$ & $2.6 \pm 0.3$ & $1.1 \pm 0.9$ & $\ldots$ \\
\hline 4068.6 & {$\left[\mathrm{~S}_{\mathrm{II}}\right]$} & $2.3 \pm 1.5$ & $2.8 \pm 0.5$ & $2.2 \pm 1.0$ & $\ldots$ & $\ldots$ & $\ldots$ & $\ldots$ & $\ldots$ \\
\hline 4101.7 & $\mathrm{H} \delta$ & $31.9 \pm 3.4$ & $32.7 \pm 1.0$ & $27.2 \pm 1.8$ & $31.9 \pm 1.5$ & $34.1 \pm 0.8$ & $33.3 \pm 0.7$ & $26.5 \pm 2.4$ & $\ldots$ \\
\hline 4200.0 & He II & $\ldots$ & $\ldots$ & & $\ldots$ & $2.3 \pm 0.2$ & $1.2 \pm 0.2$ & $\ldots$ & $\ldots$ \\
\hline 4340.5 & $\mathrm{H} \gamma$ & $54.0 \pm 3.5$ & $57.2 \pm 1.0$ & $49.9 \pm 1.8$ & $54.9 \pm 1.6$ & $56.6 \pm 1.0$ & $57.4 \pm 0.9$ & $46.2 \pm 3.3$ & $\ldots$ \\
\hline 4363.2 & [O III] & $19.6 \pm 2.2$ & $17.1 \pm 0.6$ & $10.0 \pm 1.0$ & $9.7 \pm 0.8$ & $17.0 \pm 0.6$ & $18.3 \pm 0.4$ & $16.0 \pm 1.8$ & $\ldots$ \\
\hline 4471.5 & $\mathrm{He}_{\mathrm{I}}$ & $\ldots$ & & & & thes & $3.7 \pm 0.2$ & $3.8 \pm 1.1$ & $\ldots$ \\
\hline 4540.0 & He II & $3.8 \pm 1.1$ & $4.3 \pm 0.4$ & $3.4 \pm 0.8$ & $4.1 \pm 0.7$ & $4.4 \pm 0.5$ & $2.4 \pm 0.3$ & $2.4 \pm 0.8$ & $\ldots$ \\
\hline 4686.0 & He II & $112.8 \pm 3.6$ & $116.4 \pm 1.6$ & $122.8 \pm 2.9$ & $133.0 \pm 2.5$ & $118.5 \pm 1.4$ & $65.2 \pm 0.9$ & $78.0 \pm 2.9$ & $\ldots$ \\
\hline 4711.4 & [Ar IV] & $22.6 \pm 1.8$ & $24.2 \pm 0.6$ & $20.9 \pm 1.3$ & $20.4 \pm 1.0$ & $25.2 \pm 0.6$ & $11.2 \pm 0.4$ & $14.5 \pm 1.4$ & $\ldots$ \\
\hline 4740.2 & [Ar IV] & $14.5 \pm 1.4$ & $17.7 \pm 0.6$ & $14.8 \pm 1.1$ & $14.5 \pm 0.9$ & $18.5 \pm 0.6$ & $8.1 \pm 0.4$ & $10.3 \pm 1.2$ & $\ldots$ \\
\hline 4861.4 & $\mathrm{H} \beta$ & $100 \pm 2.2$ & $100 \pm 0.9$ & $100 \pm 1.8$ & $100 \pm 1.6$ & $100 \pm 0.7$ & $100 \pm 0.5$ & $100 \pm 2.6$ & $100 \pm$ \\
\hline 4958.9 & [O III] & $305.2 \pm 2.2$ & $213.6 \pm 8.1$ & $122.4 \pm 1.0$ & $118.7 \pm 0.6$ & $210.4 \pm 9.3$ & $392.3 \pm 11.9$ & $283.1 \pm 27.2$ & $453.1 \pm 152.8$ \\
\hline 5006.8 & [O III] & $907.0 \pm 5.7$ & $638.5 \pm 5.7$ & $366.5 \pm 2.6$ & $354.2 \pm 1.5$ & $628.5 \pm 6.8$ & $1174.7 \pm 8.6$ & $849.4 \pm 27.9$ & $1130.4 \pm 419.3$ \\
\hline 5197.9 & {$\left[\mathrm{~N}_{\mathrm{I}}\right]$} & $\ldots$ & & & & $\ldots$ & $1.8 \pm 0.1$ & $1.1 \pm 0.3$ & $\ldots$ \\
\hline 5411.0 & He II & $6.2 \pm 0.7$ & $8.1 \pm 0.2$ & $7.4 \pm 0.5$ & $8.1 \pm 0.4$ & $8.3 \pm 0.2$ & $4.6 \pm 0.1$ & $4.6 \pm 0.6$ & $\ldots$ \\
\hline 5517.7 & {$[\mathrm{Cl}$ III] } & $\ldots$ & $1.0 \pm 0.1$ & $\ldots$ & $\ldots$ & $1.0 \pm 0.1$ & $1.4 \pm 0.1$ & $\ldots$ & $\ldots$ \\
\hline 5537.9 & {$[\mathrm{Cl}$ III] } & $\ldots$ & $0.7 \pm 0.1$ & $\ldots$ & $\ldots$ & $0.8 \pm 0.1$ & $1.1 \pm 0.1$ & $\ldots$ & $\ldots$ \\
\hline 5754.6 & {$\left[\mathrm{~N}_{\mathrm{II}}\right]$} & $\ldots$ & $0.4 \pm 0.1$ & $\ldots$ & $\ldots$ & $\ldots$ & $2.3 \pm 0.1$ & $2.2 \pm 0.6$ & $\ldots$ \\
\hline 5875.6 & $\mathrm{He}_{\mathrm{I}}$ & $0.9 \pm 0.2$ & $2.0 \pm 0.1$ & $1.7 \pm 0.2$ & $1.7 \pm 0.2$ & $1.8 \pm 0.1$ & $6.8 \pm 0.2$ & $4.3 \pm 0.6$ & $\ldots$ \\
\hline 6101.0 & {$\left[\mathrm{~K}_{\mathrm{IV}}\right]$} & $\ldots$ & $0.5 \pm 0.1$ & $\ldots$ & $\ldots$ & $0.7 \pm 0.1$ & $0.2 \pm 0.1$ & $0.6 \pm 0.4$ & $\ldots$ \\
\hline 6234.0 & He II & $\ldots$ & $\ldots$ & & & $\ldots$ & & $\ldots$ & $\ldots$ \\
\hline 6312.1 & [S III] & $3.8 \pm 0.4$ & $\ldots$ & $2.1 \pm 0.4$ & $1.9 \pm 0.3$ & $4.2 \pm 0.1$ & $5.6 \pm 0.1$ & $3.9 \pm 0.7$ & $\ldots$ \\
\hline 6363.0 & {$\left[\mathrm{O}_{\mathrm{I}}\right]$} & $\ldots$ & $0.4 \pm 0.1$ & $\ldots$ & $\ldots$ & $\ldots$ & $3.3 \pm 0.1$ & $1.8 \pm 0.5$ & $\ldots$ \\
\hline 6406.0 & He II & $\ldots$ & $1.1 \pm 0.2$ & & $1.0 \pm 0.5$ & $1.2 \pm 0.2$ & $0.5 \pm 0.2$ & & $\ldots$ \\
\hline 6435.1 & [Arv] & $2.1 \pm 0.6$ & $3.8 \pm 0.3$ & $5.6 \pm 0.7$ & $6.7 \pm 0.6$ & $4.0 \pm 0.2$ & $0.9 \pm 0.1$ & $1.9 \pm 0.9$ & $\ldots$ \\
\hline 6548.1 & {$[\mathrm{~N}$ II $]$} & $4.5 \pm 0.2$ & $8.3 \pm 0.1$ & $2.8 \pm 0.1$ & $1.8 \pm 0.0$ & $4.4 \pm 0.1$ & $66.2 \pm 1.1$ & $52.0 \pm 3.4$ & $\ldots$ \\
\hline 6562.8 & $\mathrm{H} \alpha$ & $287.0 \pm 5.5$ & $287.0 \pm 1.6$ & $287.0 \pm 4.1$ & $287.0 \pm 3.1$ & $287.0 \pm 1.4$ & $287.0 \pm 1.5$ & $286.5 \pm 6.4$ & $248.5 \pm 64.3$ \\
\hline 6583.5 & {$\left[\mathrm{~N}_{\mathrm{II}}\right]$} & $15.8 \pm 1.3$ & $25.0 \pm 0.5$ & $7.9 \pm 0.7$ & $6.0 \pm 0.4$ & $15.3 \pm 0.3$ & $191.6 \pm 1.2$ & $149.9 \pm 4.5$ & $\ldots$ \\
\hline 6678.2 & $\mathrm{He}_{\mathrm{I}}$ & $\ldots$ & $\ldots$ & $\ldots$ & $\ldots$ & $\ldots$ & $4.5 \pm 0.3$ & $2.4 \pm 0.9$ & $\ldots$ \\
\hline 6716.5 & {$[\mathrm{~S}$ II] } & $8.0 \pm 1.6$ & $7.9 \pm 0.4$ & $3.3 \pm 1.0$ & $2.2 \pm 0.8$ & $6.1 \pm 0.3$ & $46.0 \pm 0.8$ & $51.7 \pm 3.3$ & $\ldots$ \\
\hline 6730.8 & {$[\mathrm{~S}$ II] } & $6.6 \pm 1.5$ & $8.2 \pm 0.4$ & $2.5 \pm 0.9$ & $1.8 \pm 0.9$ & $5.9 \pm 0.3$ & $58.2 \pm 0.9$ & $50.4 \pm 3.2$ & $\ldots$ \\
\hline 7005.9 & {$[\mathrm{Arv}]$} & $15.6 \pm 4.0$ & $25.6 \pm 1.3$ & $41.9 \pm 4.0$ & $43.6 \pm 3.2$ & $29.0 \pm 1.0$ & $7.5 \pm 0.5$ & $15.4 \pm 4.5$ & $\ldots$ \\
\hline 7065.2 & $\mathrm{He}_{\mathrm{I}}$ & $\ldots$ & $2.3 \pm 0.6$ & $\ldots$ & $\ldots$ & $2.8 \pm 0.5$ & $9.2 \pm 0.5$ & $\ldots$ & $\ldots$ \\
\hline 7135.8 & {$[$ Ar III] } & $78.7 \pm 6.6$ & $84.2 \pm 1.7$ & $40.0 \pm 3.7$ & $30.0 \pm 2.4$ & $75.8 \pm 1.3$ & $158.2 \pm 1.7$ & $99.0 \pm 9.1$ & $\cdots$ \\
\hline $\log (\mathrm{H} \beta)$ & {$\left[\mathrm{erg} \mathrm{cm} \mathrm{cm}^{-2} \mathrm{~s}^{-1}\right]$} & -13.26 & -12.55 & -13.45 & -13.14 & -12.68 & -12.73 & -13.65 & -15.24 \\
\hline$c(\mathrm{H} \beta)$ & & 0.38 & 0.16 & 0.06 & 0.22 & 0.17 & 0.08 & 0 & 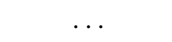 \\
\hline$A_{V}$ & & 0.80 & 0.32 & 0.13 & 0.47 & 0.35 & 0.16 & 0 & 0 \\
\hline$T_{\mathrm{e}}\left(\left[\mathrm{O}_{\mathrm{III}}\right]\right)$ & {$[\mathrm{K}]$} & $16000 \pm 800$ & $17800 \pm 200$ & $18000 \pm 900$ & $18000 \pm 800$ & $18000 \pm 200$ & $13800 \pm 100$ & $15000 \pm 500$ & $\ldots$ \\
\hline$T_{\mathrm{e}}\left(\left[\mathrm{N}_{\mathrm{II}}\right]\right)$ & {$[\mathrm{K}]$} & & $11000 \pm 1100$ & & & $\ldots$ & $9330 \pm 120$ & $10300 \pm 1100$ & $\ldots$ \\
\hline$n_{\mathrm{e}}\left(\left[\mathrm{S}_{\mathrm{II}}\right]\right)$ & {$\left[\mathrm{cm}^{-3}\right]$} & $250 \pm 60$ & $850 \pm 50$ & $100 \pm 80$ & $280 \pm 200$ & $680 \pm 40$ & $1640 \pm 40$ & $620 \pm 50$ & $\ldots$ \\
\hline$n_{\mathrm{e}}\left(\left[\mathrm{Cl}_{\mathrm{III}}\right]\right)$ & {$\left[\mathrm{cm}^{-3}\right]$} & $\ldots$ & $\ldots$ & $\ldots$ & $\ldots$ & $270 \pm 220$ & $880 \pm 80$ & $\ldots$ & $\ldots$ \\
\hline$n_{\mathrm{e}}([\mathrm{Ar} \mathrm{IV}])$ & {$\left[\mathrm{cm}^{-3}\right]$} & $\ldots$ & $420 \pm 80$ & $\ldots$ & $50 \pm 170$ & $440 \pm 90$ & $330 \pm 130$ & $100 \pm 180$ & $\cdots$ \\
\hline
\end{tabular}

respect to those of $T_{\mathrm{e}}\left(\left[\mathrm{O}_{\mathrm{III}}\right]\right)$ indicate that these apertures probe a mix of low- and high-excitation material.

As for $n_{\mathrm{e}}\left(\left[\mathrm{S}_{\mathrm{II}}\right]\right)$, it varies in Tables 3 and 4 from $100 \mathrm{~cm}^{-3}$ to $1640 \mathrm{~cm}^{-3}$, with the densest region corresponding to the core of the SW knot A7, whose head A6 and tail A8 have intermediate density values $600-700 \mathrm{~cm}^{-3}$. The NE knot seems to have lower density estimates than the SW know, but we note that Slit A does not goes exactly across the NE knot. Furthermore, the NE knot is smaller and appears more fragmented than the SW knot (see Fig. 1 right panels). These facts will have consequences for the abundance determination too (see below).

Whereas the regions A3, A5, B4 and B6 immediately around the CSPN share similar values of $T_{\mathrm{e}}$, the densities $n_{\mathrm{e}}([\mathrm{S}$ II] $])$ are larger for regions B4 and B6 $\left(n_{\mathrm{e}}=810-1100 \mathrm{~cm}^{-3}\right)$ than for regions $\mathrm{A} 3$ 
Table 4. Reddeding-corrected line intensities for selected regions of Slit B of NGC 2371

\begin{tabular}{|c|c|c|c|c|c|c|c|c|c|c|}
\hline$\lambda_{0}$ & line & $\begin{array}{c}\text { B1 } \\
\left(15^{\prime \prime}\right)\end{array}$ & $\begin{array}{c}\text { B2 } \\
\left(10^{\prime \prime}\right)\end{array}$ & $\begin{array}{c}\text { B3 } \\
\left(10^{\prime \prime}\right)\end{array}$ & $\begin{array}{c}\text { B4 } \\
\left(10^{\prime \prime}\right)\end{array}$ & $\begin{array}{c}\text { B5 } \\
\left(10^{\prime \prime}\right)\end{array}$ & $\begin{array}{c}\text { B6 } \\
\left(10^{\prime \prime}\right)\end{array}$ & $\begin{array}{c}\text { B7 } \\
\left(10^{\prime \prime}\right)\end{array}$ & $\begin{array}{c}\text { B8 } \\
\left(10^{\prime \prime}\right)\end{array}$ & $\begin{array}{c}\text { B9 } \\
\left(15^{\prime \prime}\right)\end{array}$ \\
\hline 3726.2 & {$\left[\mathrm{O}_{\mathrm{II}}\right]$} & $258.7 \pm 41.7$ & $\ldots$ & $\ldots$ & $\ldots$ & $\ldots$ & $\ldots$ & $\ldots$ & $\ldots$ & $145.9 \pm 32.4$ \\
\hline 3868.8 & [Ne III $]$ & $190.1 \pm 35.2$ & $100.6 \pm 37.2$ & $100.1 \pm 29.5$ & $36.8 \pm 3.8$ & $\ldots$ & $41.4 \pm 2.6$ & $\ldots$ & $\ldots$ & $175.8 \pm 35.9$ \\
\hline 3888.7 & $\mathrm{He}_{\mathrm{I}}$ & $\ldots$ & $\ldots$ & $\ldots$ & $12.2 \pm 3.1$ & $\ldots$ & $14.1 \pm 2.0$ & $\ldots$ & $\ldots$ & $\ldots$ \\
\hline 3967.5 & [Ne III] & $73.9 \pm 18.9$ & $\ldots$ & $\ldots$ & $29.8 \pm 3.7$ & $\ldots$ & $27.9 \pm 2.3$ & $\ldots$ & $\ldots$ & $73.3 \pm 21.6$ \\
\hline 4101.7 & $\mathrm{H} \delta$ & $31.4 \pm 11.6$ & $\ldots$ & $\ldots$ & $30.7 \pm 3.0$ & $26.6 \pm 2.4$ & $34.2 \pm 2.1$ & $38.4 \pm 13.7$ & $\ldots$ & $30.2 \pm 11.6$ \\
\hline 4340.5 & $\mathrm{H} \gamma$ & $53.0 \pm 10.8$ & $57.9 \pm 18.7$ & $36.9 \pm 12.3$ & $56.5 \pm 3.2$ & $90.0 \pm 2.9$ & $58.5 \pm 2.0$ & $51.5 \pm 14.3$ & $72.5 \pm 23.4$ & $55.7 \pm 10.7$ \\
\hline 4363.2 & [O III] & $21.6 \pm 7.7$ & $44.5 \pm 17.1$ & $\ldots$ & $11.7 \pm 2.0$ & $10.6 \pm 3.1$ & $12.0 \pm 1.2$ & $\ldots$ & $\ldots$ & $14.4 \pm 6.5$ \\
\hline 4540.0 & He II & $\ldots$ & $\ldots$ & $\ldots$ & $\ldots$ & $\ldots$ & $3.8 \pm 0.8$ & $\ldots$ & $\ldots$ & $\ldots$ \\
\hline 4686.0 & He II & $96.9 \pm 13.3$ & $107.6 \pm 19.9$ & $107.6 \pm 15.4$ & $130.8 \pm 4.0$ & $\ldots$ & $133.1 \pm 3.2$ & $126.1 \pm 12.0$ & $138.4 \pm 22.7$ & $103.3 \pm 12.9$ \\
\hline 4711.4 & [Ar IV] & $\ldots$ & $\ldots$ & $15.4 \pm 8.6$ & $22.4 \pm 2.0$ & $\ldots$ & $20.9 \pm 1.1$ & $\ldots$ & $\ldots$ & $\ldots$ \\
\hline 4740.2 & [Ar IV] & $\ldots$ & $\ldots$ & $12.6 \pm 7.9$ & $16.1 \pm 1.7$ & $14.6 \pm 1.6$ & $17.3 \pm 0.9$ & $\ldots$ & $\ldots$ & $\ldots$ \\
\hline 4861.4 & $\mathrm{H} \beta$ & $100 \pm 9.6$ & $100 \pm 16.9$ & $100 \pm 12.2$ & $100 \pm 3.4$ & $100 \pm 1.3$ & $100 \pm 1.8$ & $100 \pm 8.8$ & $100 \pm 15.6$ & $100 \pm 10.6$ \\
\hline 4958.9 & {$\left[\mathrm{O}_{\mathrm{III}}\right]$} & $533.3 \pm 21.2$ & $407.2 \pm 28.1$ & $278.2 \pm 17.7$ & $135.7 \pm 3.8$ & $154.7 \pm 1.9$ & $135.1 \pm 2.1$ & $253.5 \pm 12.5$ & $329.5 \pm 23.5$ & $478.5 \pm 22.3$ \\
\hline 5006.8 & [O III $]$ & $1549.7 \pm 31.1$ & $1191.3 \pm 45.1$ & $807.0 \pm 26.0$ & $401.9 \pm 6.0$ & $448.3 \pm 3.1$ & $394.9 \pm 3.4$ & $711.3 \pm 21.5$ & $954.6 \pm 41.3$ & $1385.8 \pm 38.6$ \\
\hline 5411.0 & $\mathrm{He}_{\text {II }}$ & $5.7 \pm 2.2$ & $\ldots$ & $\ldots$ & $6.5 \pm 0.6$ & $8.1 \pm 0.9$ & $7.5 \pm 0.4$ & $7.4 \pm 2.5$ & $\ldots$ & $5.3 \pm 2.1$ \\
\hline 5875.6 & $\mathrm{He}_{\mathrm{I}}$ & $\ldots$ & $\ldots$ & $\ldots$ & $2.0 \pm 0.4$ & $\ldots$ & $2.1 \pm 0.2$ & $\ldots$ & $\ldots$ & $\ldots$ \\
\hline 6101.0 & {$\left[\mathrm{~K}_{\mathrm{IV}}\right]$} & $\ldots$ & $\ldots$ & $\ldots$ & $\ldots$ & $\ldots$ & $\ldots$ & $\ldots$ & $\ldots$ & $\ldots$ \\
\hline 6312.1 & {$[\mathrm{~S}$ III $]$} & $5.8 \pm 2.9$ & $\ldots$ & $\ldots$ & $1.7 \pm 0.6$ & $\ldots$ & $2.0 \pm 0.3$ & $\ldots$ & $\ldots$ & $4.8 \pm 1.9$ \\
\hline 6435.1 & [Arv] & $\ldots$ & $\ldots$ & $\ldots$ & $4.3 \pm 0.9$ & $3.8 \pm 1.7$ & $4.2 \pm 0.4$ & $\ldots$ & $\ldots$ & $\ldots$ \\
\hline 6548.1 & {$[\mathrm{~N}$ II $]$} & $46.0 \pm 6.3$ & $24.5 \pm 11.1$ & $18.2 \pm 2.8$ & $3.4 \pm 0.2$ & $\ldots$ & $1.0 \pm 0.1$ & $\ldots$ & $\ldots$ & $31.8 \pm 5.1$ \\
\hline 6562.8 & $\mathrm{H} \alpha$ & $287.0 \pm 17.7$ & $287.0 \pm 26.3$ & $287.0 \pm 17.4$ & $287.0 \pm 6.8$ & $287.0 \pm 3.9$ & $287.0 \pm 4.3$ & $287.0 \pm 17.6$ & $287.0 \pm 32.2$ & $287.0 \pm 15.0$ \\
\hline 6583.5 & {$[\mathrm{~N}$ II $]$} & $159.0 \pm 13.0$ & $49.3 \pm 11.7$ & $43.0 \pm 7.0$ & $6.2 \pm 1.3$ & $12.5 \pm 2.7$ & $3.7 \pm 0.5$ & $9.4 \pm 4.1$ & $21.0 \pm 10.0$ & $106.6 \pm 9.0$ \\
\hline 6716.5 & {$[\mathrm{~S}$ II $]$} & $46.6 \pm 9.9$ & $\ldots$ & $17.4 \pm 7.9$ & $2.8 \pm 1.6$ & $2.5 \pm 1.8$ & $2.0 \pm 0.6$ & $\ldots$ & $\ldots$ & $33.6 \pm 6.6$ \\
\hline 6730.8 & {$[\mathrm{~S} \mathrm{II}]$} & $41.4 \pm 9.4$ & $\ldots$ & $18.0 \pm 8.2$ & $2.8 \pm 1.5$ & $2.9 \pm 2.3$ & $2.2 \pm 0.6$ & $\ldots$ & $\ldots$ & $31.1 \pm 6.5$ \\
\hline 7005.9 & [Arv] & $\ldots$ & $\ldots$ & $\ldots$ & $28.0 \pm 5.1$ & $23.1 \pm 4.8$ & $27.8 \pm 2.8$ & $\ldots$ & $\ldots$ & $\ldots$ \\
\hline 7135.8 & [Ar III] & $115.7 \pm 2.1$ & $127.3 \pm 55.3$ & $62.5 \pm 28.4$ & $49.5 \pm 5.9$ & $37.3 \pm 5.9$ & $28.1 \pm 2.6$ & $50.0 \pm 17.8$ & $\ldots$ & $98.9 \pm 22.5$ \\
\hline $\log (\mathrm{H} \beta)$ & {$\left[\mathrm{erg} \mathrm{cm}^{-2} \mathrm{~s}^{-1}\right]$} & -13.38 & -14.10 & -13.87 & -13.13 & -12.88 & -12.66 & -13.67 & -14.14 & -13.43 \\
\hline$c(\mathrm{H} \beta)$ & & 0.49 & 0.41 & 0.46 & 0.35 & 0.56 & 0.54 & 0.54 & 0.44 & 0.47 \\
\hline$A_{V}$ & & 1.02 & 0.86 & 0.98 & 0.74 & 1.17 & 1.14 & 1.13 & 0.91 & 0.99 \\
\hline$T_{\mathrm{e}}([\mathrm{O} \mathrm{III}])$ & {$[\mathrm{K}]$} & $13100 \pm 1700$ & $22000 \pm 5000$ & $\ldots$ & $18600 \pm 1600$ & $15100 \pm 2300$ & $19000 \pm 1000$ & $\ldots$ & $\ldots$ & $11800 \pm 1800$ \\
\hline$n_{\mathrm{e}}([\mathrm{S} \mathrm{II}])$ & {$\left[\mathrm{cm}^{-3}\right]$} & $380 \pm 30$ & 然 & 690: & $810 \pm 360$ & $1190 \pm 240$ & $1070 \pm 30$ & $\ldots$ & $\ldots$ & $450 \pm 30$ \\
\hline$n_{\mathrm{e}}([\mathrm{Ar} \mathrm{IV}])$ & {$\left[\mathrm{cm}^{-3}\right]$} & $\ldots$ & $\ldots$ & $1600 \pm 500$ & $140 \pm 200$ & $\ldots$ & $2160 \pm 40$ & $\ldots$ & $\ldots$ & $\ldots$ \\
\hline
\end{tabular}

and A5 $\left(100-280 \mathrm{~cm}^{-3}\right)$. The latter seem to probe a hollow region around the CSPN. Regions B1 and B9 extracted from the outer edges of the major axis of NGC 2371 have the lowest temperature values $\left(T_{\mathrm{e}}\left(\left[\mathrm{O}_{\mathrm{III}}\right]\right) \simeq 11,800-13,100 \mathrm{~K}\right)$, but they have higher densities $\left(n_{\mathrm{e}}\left(\left[\mathrm{S}_{\mathrm{II}}\right]\right) \approx 400 \mathrm{~cm}^{-3}\right)$ than regions within the inner main cavity.

In order to unveil differences in excitation from different morphological features in NGC 2371, we plot in Figure 8 line ratios of key emission lines derived from the INT IDS spectroscopy (see Akras \& Gonçalves 2016, and references therein), including [N $\mathrm{NI}] 6583 / \mathrm{H} \alpha$ vs. [O III] 5007/H $\alpha$ (top panel) and [S II] $(6717+6731) / \mathrm{H} \alpha$ vs. [O III] 5007/H $\alpha$ (bottom panel). Differences in excitation are clear: regions close to the star (inner regions

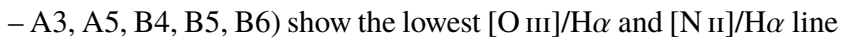
ratios, whereas other regions, particularly the outermost regions $\mathrm{B} 1$ and $\mathrm{B} 9$, and the regions $\mathrm{A} 7$ and $\mathrm{A} 8$ of the SW knot show the highest $\left[\mathrm{O}{ }_{\mathrm{III}}\right] / \mathrm{H} \alpha$ and $\left[\mathrm{N}_{\mathrm{II}}\right] / \mathrm{H} \alpha$.

In Figure 8 bottom panel we show the theoretical limit between photoionised and shocked material $\log \left(\left[\mathrm{O}_{\mathrm{III}}\right] / \mathrm{H} \alpha\right)=$ $1.89 \log ([\mathrm{S} \mathrm{II}] / \mathrm{H} \alpha)+2.46$, as estimated by Danehkar et al. (2018) based on the models presented by Raga et al. (2008). Line ratios below this limit show gas excited by ionisation, while regions above present shocked excited gas. Most morphological features in NGC 2371 are located below this theoretical limit, i.e., they are excited by photoionisation. On the other hand, the outermost B1 and
B9 regions are located above the dashed line, suggesting that they are excited by shocks as could be expected if these structures were expanding at high speed into the interstellar medium. The spectra of regions A7 and A8 extracted from the head of the SW dense knot also present line intensity ratios typical of shock-excitation.

The HST WFC3 images of NGC 2371 have also been used to provide a complementary view of its ionisation structure. Ratio maps were obtained by dividing the $\left[\mathrm{O}_{\mathrm{III}}\right],\left[\mathrm{N}\right.$ II] and [S $\left.\mathrm{S}_{\mathrm{II}}\right]$ calibrated images by the $\mathrm{H} \alpha$ image (see Fig. 9). We note that the HST [ $\left.\mathrm{S}_{\mathrm{II}}\right]$ image does not have adequate signal-to-noise ratio in the NW and SE lobes of NGC 2371. Thus, the line ratio calculations were only performed for the innermost regions of NGC 2371. We defined four different rectangular regions (see Fig. 9 left panel) to study the dominant excitation mechanisms: a large region that includes most of the main inner cavity of NGC 2371, two regions covering the SW and NE dense knots, and a region that includes only gas around the central star. We computed the pixel-by-pixel line ratios and these are plotted in Figure 8 as colour-coded regions alongside the results found for regions extracted from Slit A and B. The results are consistent with those obtained from the spectroscopic observations.

Finally, the abundances were computed using the extensively tested code PyNEB developed by Luridiana et al. (2015). PyNEB computes the physical conditions $\left(T_{\mathrm{e}}\right.$ and $\left.n_{\mathrm{e}}\right)$ and ionic and total abundances. Ionic abundances were calculated with their corre- 

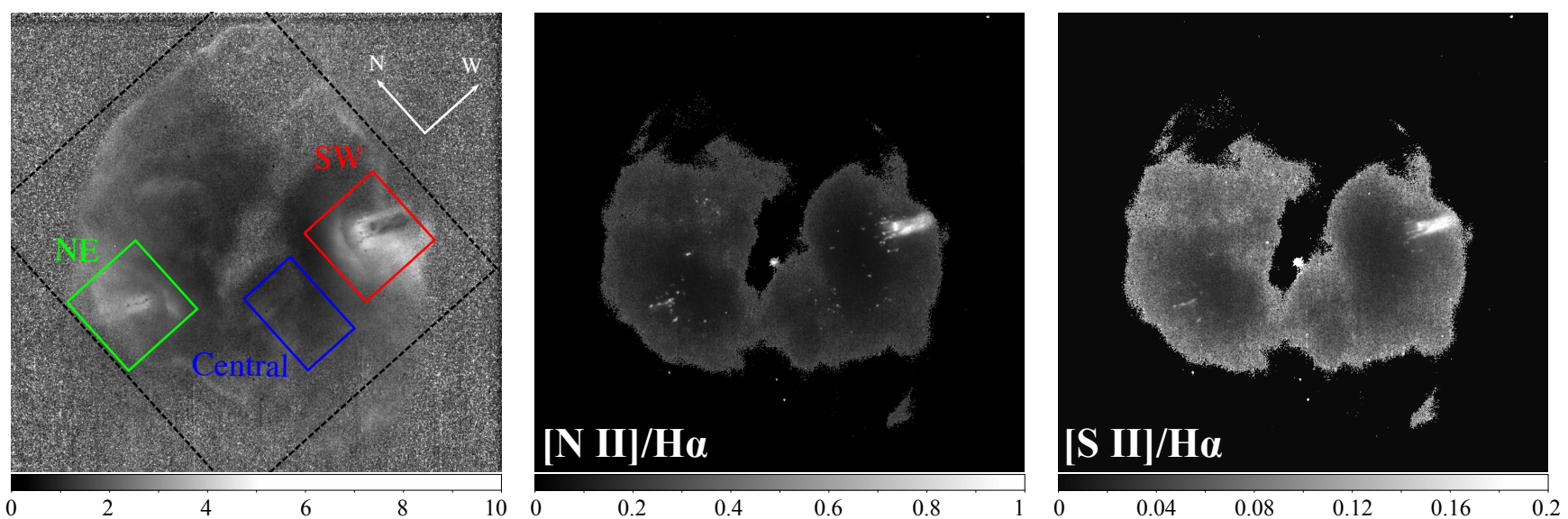

Figure 9. Line intensity ratio maps obtained from calibrated HST images. (Left) The (black) dashed-line, red line, blue line, and green line rectangles represent different regions used to study the ionisation structure of NGC 2371 in Figure 8. The three panels have the same field of view and orientation.

sponding temperature and averaged density based on the ionization potential (IP) of the ion. We note that the $T_{\mathrm{e}}$ and $n_{\mathrm{e}}$ values obtained from PyNEB (not presented here) are consistent within $<2 \%$ with those listed in Tables 3 and 4 obtained in IRAF. For the calculation of ionic abundances, we only considered emission lines with uncertainties smaller than $50 \%$, resulting in abundances with typical uncertainties of a few percent. The total abundances of $\mathrm{He}, \mathrm{O}, \mathrm{N}$, $\mathrm{Ne}, \mathrm{Ar}, \mathrm{S}$ and $\mathrm{Cl}$, listed in Table 5 for apertures A1-A3 and A5-A8, were computed adopting the ionization correction factors (ICFs) provided by Delgado-Inglada et al. (2014). The error budget of the abundances of heavy elements in Table 5 is mostly dominated by uncertainties in the computation of those ICFs, which are notably large for high excitation nebulae.

\section{DISCUSSION}

NGC 2371 has a multi-component morphology with (1) a pair of outer lobes aligned along $\mathrm{PA}=128^{\circ},(2)$ a high-excitation main shell with apparent elliptical morphology aligned along the same direction with two lower excitation caps at its tips, and (3) two ensembles of dense low-ionisation knots in the equatorial region of the main shell, although misaligned with the previous structures. We provide next a description of the overall physical structure of NGC 2371 using kinematical information to help us interpret its different components.

\subsection{On the kinematic structure of NGC 2371}

By Gaussian fitting of bright emission lines, their centroid can be routinely determined with an accuracy $\sim 10$ times better than the spectral resolution, which is $\sim 200 \mathrm{~km} \mathrm{~s}^{-1}$ for our observations with the R400V grating and $\sim 70 \mathrm{~km} \mathrm{~s}^{-1}$ for those with the R1200U grating. Thus, our low resolution spectra allow us to investigate the kinematics of structures that have velocities $\geqslant 20 \mathrm{~km} \mathrm{~s}^{-1}$. This is illustrated in Figure 10, which presents three sections of the R400V 2D-spectrum obtained with the Slit B along the major nebular axis. These spectral sections include the $\mathrm{H} \alpha+\left[\mathrm{N}_{\mathrm{II}}\right]$, [O $\left.\mathrm{O}_{\mathrm{II}}\right]$ and $[\mathrm{O} \mathrm{III}]$ emission lines. Velocity gradients are clearly seen in these (as well as other) spectral lines as an S-shaped pattern in the

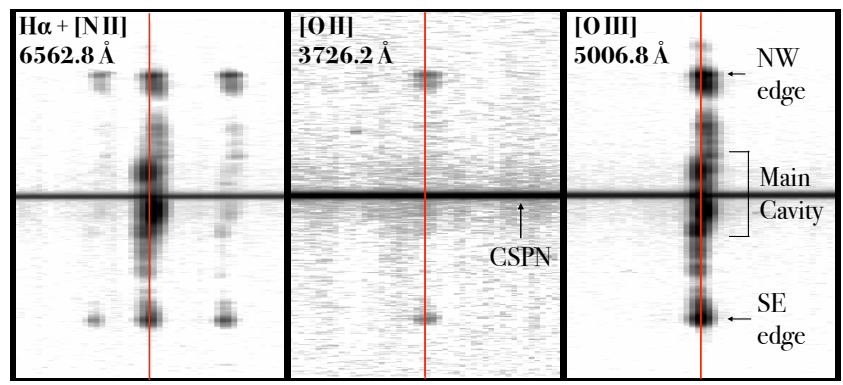

Figure 10. Sections of the INT IDS spectrum of the major axis of NGC 2371 (Slit B) centered on the $\left.\mathrm{H} \alpha+\left[\mathrm{N}_{\mathrm{II}}\right], \mathrm{O}_{\mathrm{II}}\right]$ and [O $\left.\mathrm{O}_{\mathrm{II}}\right]$ lines. The red vertical lines show the position of the rest wavelength of each line. The horizontal axis represents wavelength while the vertical the spatial profile.

position-velocity (PV) map along the nebular axis, but tilted along the opposite direction for the main cavity.

These PV maps have been used to estimate the systemic radial velocities of the different morphological features in NGC 2371 (i.e., the difference of the radial velocities of a feature with respect to the average radial velocity of the nebula) labeled in Figure 2 next to their corresponding extraction regions. The outermost structures B1 and $\mathrm{B} 9$ have systemic radial velocities of $\pm 30 \mathrm{~km} \mathrm{~s}^{-1}$, but the tips of the inner cavity B3 and B7 have larger systemic radial velocities of $\pm 70 \mathrm{~km} \mathrm{~s}^{-1}$. Meanwhile, regions B4 and B6 inside the main nebular shell have systemic radial velocities of $\pm 40 \mathrm{~km} \mathrm{~s}^{-1}$ with opposite sign than those of the adjacent tips B3 and B7. The fast expansion of the bipolar lobes in NGC 2371 is consistent with the faster expansion of PNe with [WR]-type CSPNe compared to other PNe (Peña et al. 2003).

The overall expansion patterns described above are consistent with those reported by Ayala et al. (2005) using longslit highdispersion echelle data. With that information at hand, we can envisage the simple model of the physical structure along the main nebular axis of NGC 2371 sketched in Figure 11. The physical structure of NGC 2371 shares many similarities with that of NGC 650-1 (see Ramos-Larios et al. 2018), with two pairs of bipolar lobes tilted with the line of sight along different inclination angles, and a toroidal or barrel-like central cavity. In this geometrical model, 


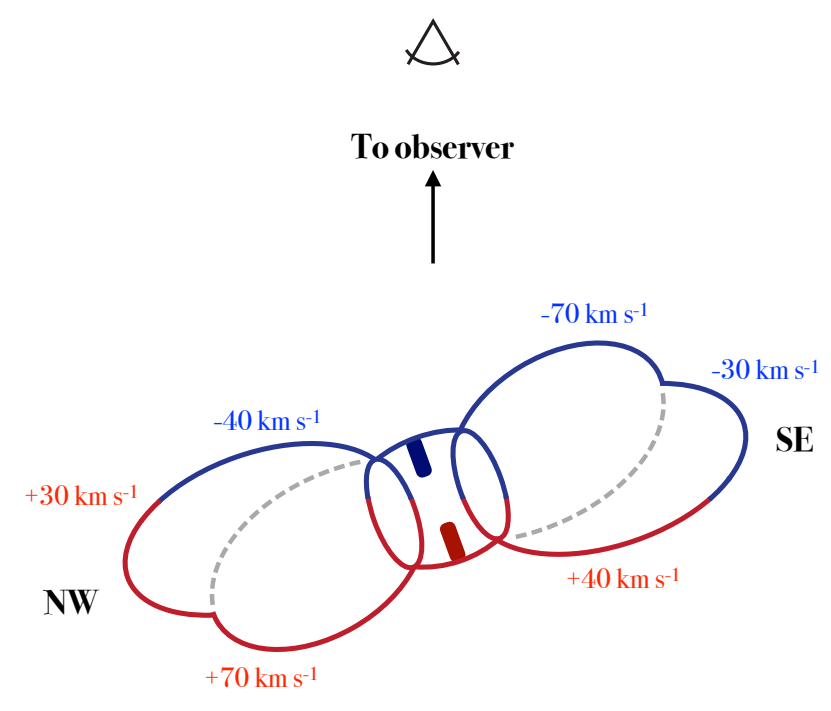

Figure 11. Velocity structure of NGC 2371 suggested by the radial velocities obtained from our spectral analysis.

the reversal of systemic radial velocities between the toroidal structure and the tips of the innermost bipolar lobes B3 and B7 can be explained if the toroidal structure is orthogonal to the bipolar lobes.

The present data allow a basic description of NGC 2371, but we note that the NW and SE lobes have a rich morphology that suggests a more complex structure than that proposed in Figure 11. A complete analysis of the physical structure of NGC 2371 using information obtained along different slit positions using the Manchester Echelle Spectrograph (MES) at the $2.1 \mathrm{~m}$ telescope of the Observatorio Astronómico Nacional de San Pedro Mártir (SPM-OAN) in conjunction with a morpho-kinematic model is underway (Vázquez et al., in preparation) and will certainly produce a detailed view of the physical structure of this PN to peer further into its formation history. Yet, the available data still have an interesting piece of information. The NE and SW low-ionisation knots move at systemic radial velocities $\pm 7 \mathrm{~km} \mathrm{~s}^{-1}$ considerably smaller than that of the toroidal structure of the main nebula where they seem to be embedded. These velocities, which are consistent with those reported by Sabbadin et al. (1982), imply that the knots are being overtaken by the nebular material as suggested by Ramos-Larios \& Phillips (2012). Furthermore, the arc-like features observed around these structures (see Fig. 1 right panels) seems to suggest that they are interacting with the current fast wind from the CSPN, similarly to what is observed in HST images of Orion proplyds (see GarcíaArredondo et al. 2001).

\subsection{A comprehensive view of NGC 2371}

The spatio-kinematic model of NGC 2371 described above can be compared to its excitation structure and spatially varying chemical abundances. Despite its complex morphology, NGC 2371 is quite symmetric, with an ellipsoidal main nebular shell with polar blowouts and a pair of bipolar lobes whose symmetry axis is aligned along a slightly tilted direction. The tips of the bipolar lobes show clear evidence of shock-excitation, which can arise as the nebular material expands at relatively high speed and interacts with the
ISM. On the other hand, the main nebular shell and very notably the regions close to the CSPN exhibit very high excitation, with the lowest $[\mathrm{N} \mathrm{II}] / \mathrm{H} \alpha$ and highest $\mathrm{He} \mathrm{II} / \mathrm{H} \beta$ line ratios. Indeed, excitation in these innermost regions is so high that the $[\mathrm{O} \mathrm{III}] / \mathrm{H} \alpha$ line ratio is damped as $\mathrm{O}^{++}$is photo-ionised to higher ionisation levels. Furthermore, several He II emission lines can be seen in the optical spectrum shown in Figure 5. Along with $\mathrm{C}$ IV and [Ne IV], it has also been reported to be present in the IUE UV nebular spectrum of NGC 2371 (see fig. 2 in Pottasch et al. 1981). Since the He II $\lambda 1640 \AA$ is the dominant line in the wavelength range between 1400 $\AA$ and $1800 \AA$, the GALEX far-UV image in Figure 12 (left panel) can be used to probe the spatial location of high-excitation material in NGC 2371. The emission in this image traces the main morphological features of NGC 2371, including the main cavity, the dense knots, and the outer edges of the NW and SE lobes. In addition, there is a diffuse halo that extends to distances of $\sim 70$ arcsec from the CSPN, which is not detected in the near-UV GALEX image (see Fig. 12 right panel). When comparing with mid-IR Spitzer presented in Ramos-Larios \& Phillips (2012) and optical CFHT [O III] images, there is notable emission in the Spitzer images close to the CSPN, most likely associated with high-excitation [O IV] emission lines. The high excitation of the inner regions of NGC 2371 results from the strong UV flux of WD $0722+295$, whereas the high-excitation of the outer halo might result from the "hardening" of the stellar radiation, as high energy photons leak from the main nebular shell of NGC 2371 (see Guerrero \& Manchado 1999; Gesicki et al. 2003).

The low-ionisation dense SW and NE knots are aligned along a direction different from the main nebular axis and they do not share the expansion velocity of the main nebula, with lower expansion velocities $\left( \pm 7 \mathrm{~km} \mathrm{~s}^{-1}\right)$. Their morphology, with bow-shocks pointing towards the ionisation source, also precludes a jet interpretation for these features, but they are more consistent with clumps surrounded by photo-evaporated material. Thus, whereas the main nebula of NGC 2371 has very high-excitation, it seems that the face of these knots pointing towards the CSPN shields their cometary tails from the UV stellar flux, producing emission from low ionisation species at their tails. Indeed, the spectra extracted from these structures present emission from [O $\left.\mathrm{O}_{\mathrm{I}}\right]$ and $\left[\mathrm{N}_{\mathrm{I}}\right]$ (see $\mathrm{A} 2, \mathrm{~A} 7$ and A8 in Tab. 2) suggesting them to be low-ionisation structures (LIS). The analysis of LIS in PNe presented by Akras \& Gonçalves (2016) suggests $T_{\mathrm{e}}=10,000-14,700 \mathrm{~K}$, consistent with our estimates for the SW and NE knots, but lower electron densities than the surrounding ionised gas, contrary to the SW and NE knots of NGC 2371, which are much denser than the nebular material where they are embedded (see Tab. 2). In this sense, the SW and NE knots of NGC 2371 are not typical LIS.

The chemical abundances of different apertures along the minor axis of NGC 2371 are listed in Table 5. The mean value of the He abundances is $0.113 \pm 0.012(\simeq 1.4$ times the solar value in Lodders 2010), with most individual values within $1-\sigma$ uncertainty. This might also be the case for the $\mathrm{O}$ abundances, with a mean value of $(4.1 \pm 2.3) \times 10^{-4}$, which is mostly consistent within $1-\sigma$ uncertainty with that of individual apertures. This mean value suggests slightly subsolar $\mathrm{O}$ abundances, $(\mathrm{O} / \mathrm{H}) \simeq 0.75(\mathrm{O} / \mathrm{H})_{\odot}$. On the contrary, the $\mathrm{N}$ abundances are clearly larger than solar, with a mean N/O ratio of $0.38 \pm 0.11$, i.e., $(\mathrm{N} / \mathrm{O}) \approx 3(\mathrm{~N} / \mathrm{O})_{\odot}$. The chemical abundances $(\mathrm{He} / \mathrm{H}, \mathrm{O} / \mathrm{H}, \mathrm{N} / \mathrm{O})$ of NGC 2371 are basically consistent with those reported for comprehensive samples of PNe with [WC] central stars (Peña et al. 2001; García-Rojas et al. 2013). The other chemical abundances in Table 5 have larger uncertainties, particularly the Ar abundances, for which no error bar is provided. The values of the 

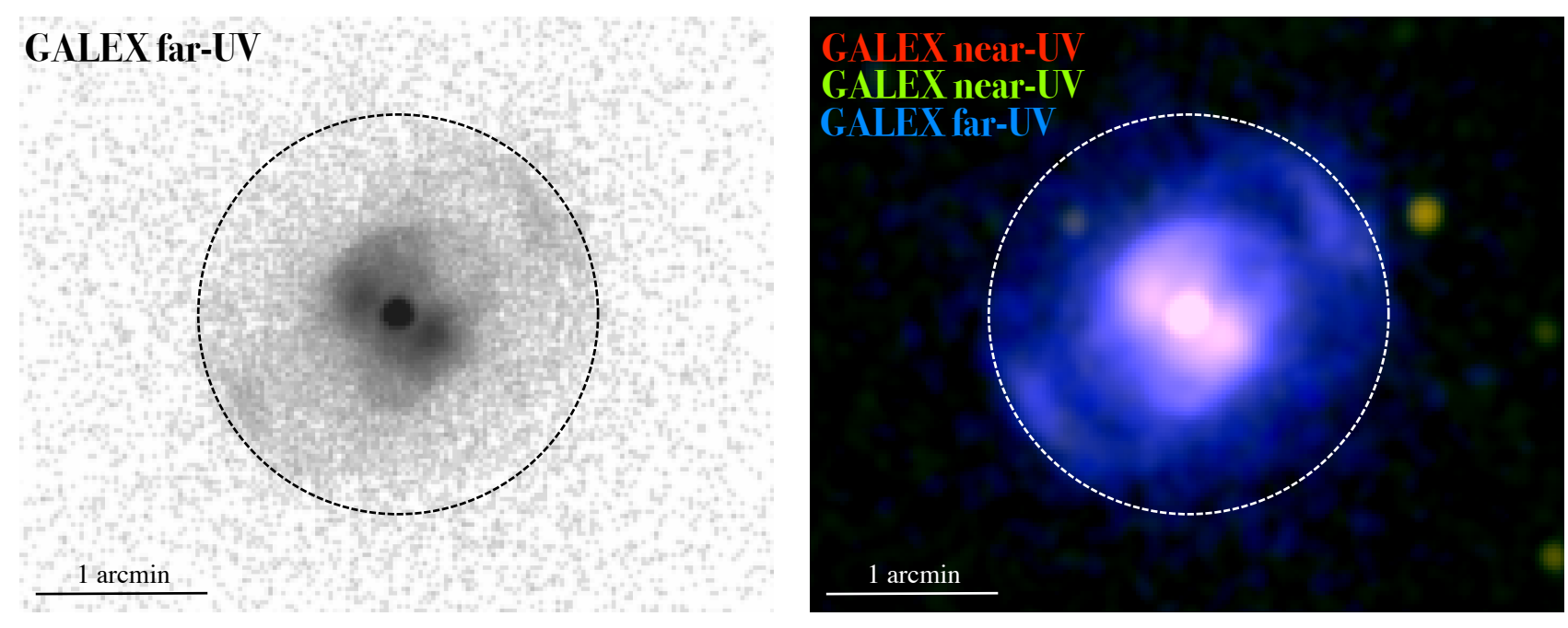

Figure 12. GALEX UV images of NGC 2371. (Left) GALEX far-UV gray scale image with the natural GALEX pixel size of 1.5 arcsec. (Right) Colour-composite UV image using the near- and far-UV filters. The dashed-line circle in both panels has an angular radius of 70 arcsec. North is up and east to the left.

Table 5. Total abundances for different regions in NGC 2371

\begin{tabular}{ccccccccc}
\hline & A1 & A2 & A3 & A5 & A6 & A7 & A8 & $\begin{array}{c}\text { Solar } \\
\text { (Lodders 2010) }\end{array}$ \\
\hline $\mathrm{He}$ & $0.106 \pm 0.013$ & $0.116 \pm 0.005$ & $0.122 \pm 0.005$ & $0.130 \pm 0.006$ & $0.118 \pm 0.003$ & $0.102 \pm 0.002$ & $0.098 \pm 0.007$ & 0.084 \\
$\mathrm{O}$ & $(7.1 \pm 3.0) \times 10^{-4}$ & $(3.8 \pm 1.3) \times 10^{-4}$ & $(1.6 \pm 0.6) \times 10^{-4}$ & $(1.7 \pm 0.7) \times 10^{-4}$ & $(2.8 \pm 0.9) \times 10^{-4}$ & $(6.8 \pm 2.4) \times 10^{-4}$ & $(4.9 \pm 1.7) \times 10^{-4}$ & $5.37 \times 10^{-4}$ \\
$\mathrm{~N}$ & $(2.2 \pm 1.4) \times 10^{-4}$ & $(1.1 \pm 0.4) \times 10^{-4}$ & $(9.2 \pm 3.2) \times 10^{-5}$ & $(4.7 \pm 1.7) \times 10^{-5}$ & $(1.3 \pm 0.4) \times 10^{-4}$ & $(2.2 \pm 0.8) \times 10^{-4}$ & $(2.1 \pm 0.7) \times 10^{-4}$ & $7.24 \times 10^{-5}$ \\
$\mathrm{Ne}$ & $(3.4 \pm 1.4) \times 10^{-4}$ & $(3.4 \pm 1.0) \times 10^{-4}$ & $(8.1 \pm 2.4) \times 10^{-5}$ & $(1.1 \pm 0.4) \times 10^{-4}$ & $(1.6 \pm 0.5) \times 10^{-4}$ & $(1.4 \pm 0.4) \times 10^{-5}$ & $(1.8 \pm 0.5) \times 10^{-4}$ & $1.12 \times 10^{-4}$ \\
$\mathrm{Ar}$ & $3.5 \times 10^{-5}:$ & $1.5 \times 10^{-5}:$ & $1.1 \times 10^{-5}$ & $5.0 \times 10^{-6}:$ & $1.2 \times 10^{-5}:$ & $1.3 \times 10^{-5}:$ & $9.3 \times 10^{-6}:$ & $3.16 \times 10^{-6}$ \\
$\mathrm{~S}$ & $(2.9 \pm 1.5) \times 10^{-5}$ & $(7.2 \pm 2.9) \times 10^{-6}$ & $(8.5 \pm 3.4) \times 10^{-6}$ & $(4.4 \pm 1.8) \times 10^{-6}$ & $(1.5 \pm 0.6) \times 10^{-5}$ & $(1.7 \pm 0.7) \times 10^{-5}$ & $(1.7 \pm 0.7) \times 10^{-5}$ & $1.45 \times 10^{-5}$ \\
$\mathrm{Cl}$ & $\ldots$ & $(1.9 \pm 0.7) \times 10^{-7}$ & $\ldots$ & $\ldots$ & $(3.6 \pm 1.4) \times 10^{-7}$ & $(1.5 \pm 0.6) \times 10^{-7}$ & $\ldots$ & $1.78 \times 10^{-7}$ \\
\hline $\mathrm{He} / \mathrm{He} \odot$ & 1.26 & 1.38 & 1.45 & 1.55 & 1.41 & 1.21 & 1.17 & $\ldots$ \\
$\mathrm{N} / \mathrm{O}$ & 0.31 & 0.29 & 0.58 & 0.28 & 0.46 & 0.32 & 0.43 & 0.13 \\
$\mathrm{Ne} / \mathrm{O}$ & 0.48 & 0.89 & 0.51 & 0.65 & 0.57 & 0.21 & 0.37 & 0.21 \\
$\mathrm{Ar} / \mathrm{O}$ & $0.05:$ & $0.04:$ & $0.05:$ & $0.03:$ & $0.04:$ & $0.02:$ & $0.02:$ & 0.006 \\
$\mathrm{~S} / \mathrm{O}$ & 0.04 & 0.02 & 0.05 & 0.03 & 0.05 & 0.03 & 0.03 & 0.03 \\
\hline
\end{tabular}

chemical abundances of $\mathrm{Ne}, \mathrm{Ar}, \mathrm{S}$, and $\mathrm{Cl}$ are also consistent with those of PNe with [WC] central stars.

The chemical abundances found in different regions are in general consistent among them, within $2 \sigma$ up most from their mean values, but we note that the abundances of the main elements $\mathrm{He}$, $\mathrm{N}$, and $\mathrm{O}$ show some differences from one region to another. It might be argued that the $\mathrm{O}$ abundances from apertures probing the dense low-ionisation structures are higher than those of the main cavity enclosing them, whereas the N/O ratios are lower. We believe this is not the case, because a close inspection reveals that the $\mathrm{O}$ abundances are mostly anti-correlated with $T_{\mathrm{e}}$, underlying the difficulties to determine the chemical abundances in highly excited nebulae and from regions where low- and high-excitation material coexists.

Finally, we would like to remark that Herald \& Bianchi (2004) suggested WD $0722+295$ to be in the same evolutionary stage as the CSPN of the born-again PN A 78, a [WR]-PG 1159 star. Although their analysis of the stellar atmospheres of those two CSPNe with their version of the CMFGEN code resulted in similar atmospheres, our best-fit models of WD $0722+295$ and our recent analysis of the CSPN of A 78 (see Toala et al. 2015) show these two stars to be different. Moreover, the presence of hydrogen-deficient material inside A 78 suggests a different evolutionary path compared to the CSPN of NGC 2371. The spectral analysis of WD $0722+295$ presented here represents an improvement over other similar studies due to the high-quality of the optical spectrum and the combination with available UV data used for the fit.

The PoWR model presented here is not able to reproduce the lines identified in Section 3 at $\approx 5665 \AA$ and $\approx 6066 \AA$ as O VII $\lambda 5666$ and $\mathrm{O}$ VIII $\lambda 6068$, because the ionisation potentials of these species, $739.3 \mathrm{eV}$ and $871.4 \mathrm{eV}$, respectively, are too high to be produced by the stellar atmosphere. Although it can be argued that such high-temperature gas can be produced by the X-ray emission of WD 0722+296 detected by Chandra (Montez et al. 2015), it has been long discussed whether these emission lines are due to oxygen, but to neon. Werner et al. (2007) presented stellar atmosphere models of pre-WD stars and demonstrated that most of the emission lines from the $\mathrm{O}$ VII and $\mathrm{O}$ VIII high-ionisation states of oxygen might be 
misidentified with those of Ne VII and Ne VIII, whose ionisation potentials, $207.3 \mathrm{eV}$ and $239.1 \mathrm{eV}$, respectively, are considerably lower. This does not affect the spectral classification of WD $0722+296$ as part of the [WO] sequence, because the O vi $\lambda 3820$, O vi $\lambda 5290$, and $\mathrm{O}_{\text {VI }} \lambda 6200$ lines are still present in its spectrum (see Table 1). Indeed, the Ne viI and $\mathrm{Ne}$ viII lines require a high temperature for the central star.

\section{SUMMARY}

We presented spatially-resolved longslit INT IDS spectroscopic observations of NGC 2371, a PN with a [WR]-type CSPN with an apparent complex morphology. Our spectral observations, in conjunction with HST, GALEX and CFHT images, have allowed us to characterise the central star of NGC 2371 as well as to unveil its high-ionisation structure. Our findings can be summarised as follows:

- We studied the spectral properties of the CSPN of NGC 2371, WD $0722+295$, by analysing the IDS observations. A Gaussianfitting procedure was performed to measure the true contribution of the WR spectral lines and to assess the spectral sub-type of the CSPN. Line ratios of WR features with that of the RB suggest a [WO1]. In combination with the UV data we used the stellar atmosphere code PoWR to estimate the stellar parameters. Although we found very similar results as those obtained in previous works, the differences are due to the assumed clumping factor and a more accurate Gaia distance.

- We studied the physical properties of different regions of NGC 2371. In accordance with previous works on NGC 2371 the electron density ranges between $100 \mathrm{~cm}^{-3}$ and $1,640 \mathrm{~cm}^{-4}$. The densest regions are the SW and NE low-ionisation knots. The electron temperatures vary from $T_{\mathrm{e}} \sim 12,000 \mathrm{~K}$ at the outer edges to $T_{\mathrm{e}} \approx 18,000 \mathrm{~K}$ for regions close to the star. The outer bipolar (NW and SE) lobes have electron densities $n_{\mathrm{e}} \approx 400 \mathrm{~cm}^{-3}$ and $T_{\mathrm{e}} \approx 12,000-13,000 \mathrm{~K}$.

- The analysis of emission line ratios from the IDS spectra as well as those extracted from the HST calibrated images demonstrate the powerful effect of the UV flux from the CSPN of NGC 2371. Most emission from this $\mathrm{PNe}$ is dominated by ionisation, but the low-ionisation SW and NE knots and the outer regions B1 and B9, where shock-excitation is important. We suggest that B1 and B9 are shock-excited because they trace the shock due to the expansion of NGC 2371.

- Inspection of GALEX images unveiled the presence of a halo extended to 70 arcsec from WD $0722+295$. The halo is detected in the far-UV channel and is mainly dominated by He II emission. We suggest that this halo has formed due to high-energy photon leakage.

- We found that the bow shock-like structure around the CSPNfacing head of the low-ionisation dense knots correspond to photoevaporation flows. The head of the knots shield their tails producing emission from low-ionisation species such as $\left[\mathrm{O}_{\mathrm{I}}\right]$ and $\left[\mathrm{N}_{\mathrm{I}}\right]$. This reinforces the idea proposed by Herald \& Bianchi (2004) and Ramos-Larios \& Phillips (2012) that molecular material might be present in the vicinity of the CSPN of NGC 2371, probably in these structures similar to LIS in other PNe (Akras et al. 2017). However, we note that these clumps do not share the same physical properties as classic LIS described in the literature.

- We estimated the chemical abundances for different regions of NGC 2371. The abundances are typical of PNe with [WC] central stars. Although some variations may exist among different regions, the abundances of the dense knots lay within those of adjacent regions, suggesting that they were not ejected as the result of a VLTP. Instead, their kinematics and detailed morphology suggest they were ejected before the formation of the main nebular shell.

- The relatively high-resolution of the optical longslit spectra presented here allowed us to suggest a possible kinematic structure of NGC 2371. The outer lobes have the typical expansion velocity of evolved $\mathrm{PNe}\left(\sim 30 \mathrm{~km} \mathrm{~s}^{-1}\right)$ whilst the inner shell has an expansion velocity of $\sim 70 \mathrm{~km} \mathrm{~s}^{-1}$ in accordance with previous analysis of highresolution echelle observations. On the other hand, the dense knots have a slow radial velocity. We suggest that NGC 2371 has a bipolar shape with each lobe presenting a double-structure protruding from a barrel-like central region.

We propose that the densest material around WD 0722+295 might be the relic of an early ejection of material along a ring-like structure. The ejection of the main nebula is more recent and can be directly related to the current fast stellar wind of the progenitor star of NGC 2371. It seems to have expanded towards the low-density region, the polar zone of the barrel-like structure. We emphasise that the analysis of high-resolution echelle observations such as those obtained with the San Pedro Mártir MES are most needed in order to construct a detailed view of the morpho-kinematic structure of NGC 2371.

\section{ACKNOWLEDGEMENTS}

The authors thank the referee for a critical reading of the manuscript and valuable suggestions that improved the presentation of the paper. The authors would like to thank V. Gómez Llanos Sandoval for helping them in using PyNEB. VMAGG, JAT, MAG and HT are funded by UNAM DGAPA PAPIIT project IA100318. GRL acknowledges support from Fundación Marcos Moshinsky, CONACyT and PRODEP (Mexico). MAG acknowledges support of the Spanish Ministerio de Ciencia, Innovación y Universidades grant PGC2018-102184-B-I00, co-funded by FEDER funds. LS thanks support from UNAM PAPIIT grant IN101819. YDM thanks CONACyT for the research grant CB-A1-S-25070. This work has make extensive use of the NASA's Astrophysics Data System. This paper also presents data obtained with the MOS camera at the CanadaFrance-Hawaii Telescope (CFHT) which is operated by the National Research Council (NRC) of Canada, the Institut National des Sciences de l'Univers of the Centre National de la Recherche Scientifique of France, and the University of Hawaii. The authors thank E. Santamaría for helping produce the velocity sketch of NGC 2371. Based on observations made with the NASA/ESA Hubble Space Telescope, obtained at the Space Telescope Science Institute, which is operated by the Association of Universities for Research in Astronomy, Inc., under NASA contract NAS 5-26555.

\section{REFERENCES}

Acker, A., \& Neiner, C. 2003, A\&A, 403, 659

Acker, A., Górny, S. K., \& Stenholm, B. 1996, Ap\&SS, 238, 63

Akashi, M., \& Soker, N. 2017, MNRAS, 469, 3296

Akras, S., \& Gonçalves, D. R. 2016, MNRAS, 455, 930

Akras, S., Gonçalves, D. R., \& Ramos-Larios, G. 2017, MNRAS, 465, 1289

Aller, L. H., \& Czyzak, S. J. 1979, Ap\&SS, 62, 397

Ayala, S., Vázquez, R., Miranda, L. F., et al. 2005, Planetary Nebulae as Astronomical Tools, 95

Bailer-Jones, C. A. L., Rybizki, J., Fouesneau, M., et al. 2018, AJ, 156, 58

Balick, B. 1987, AJ, 94, 671 
Cardelli, J. A. A., Clayton, G. C., Mathis, J. S. 1989, ApJ, 345, 245

Crowther, P. A., De Marco, O., \& Barlow, M. J. 1998, MNRAS, 296, 367

Curtis, H. D. 1918, Publications of Lick Observatory, 13, 55

Danehkar, A., Karovska, M., Maksym, W. P., et al. 2018, ApJ, 852, 87

De Marco, O., \& Crowther, P. A. 1999, MNRAS, 306, 931

Delgado-Inglada, G., Morisset, C. \& Stasińska, G. 2014, MNRAS, 440, 536-554

García-Arredondo, F., Henney, W. J., \& Arthur, S. J. 2001, ApJ, 561, 830

García-Rojas, J., Peña, M., Morisset, C., et al. 2013, A\&A, 558, A122

Gesicki, K., Zijlstra, A. A., Acker, A., et al. 2006, A\&A, 451, 925

Gesicki, K., Acker, A., \& Zijlstra, A. A. 2003, A\&A, 400, 957

Gómez-González, V. M. A., Mayya, Y. D., Rosa-González, D., RodríguezMerino, L. H., Toalá, J. A. and Alvarez, C. 2020, 493, 3879

Górny, S. K. 2001, Ap\&SS, 275, 67

Górny, S. K., \& Tylenda, R. 2000, A\&A, 362, 1008

Gräfener, G., Koesterke, L., \& Hamann, W.-R. 2002, A\&A, 387, 244

Guerrero, M. A., Fang, X., Miller Bertolami, M. M., et al. 2018, Nature Astronomy, 2, 784

Guerrero, M. A., \& Manchado, A. 1999, ApJ, 522, 378

Hamann, W.-R., \& Gräfener, G. 2004, A\&A, 427, 697

Heap, S. R. 1982, IAU Symposium 99, Wolf-rayet Stars: Observations, Physics, Evolution, 423, eds. C.W.H. De Loore, and A.J. Willis

Henry, R. B. C., Stephenson, B. G., Miller Bertolami, M. M., et al. 2018, MNRAS, 473, 241

Herald, J. E., \& Bianchi, L. 2004, ApJ, 609, 378

Herwig, F., Blöcker, T., Langer, N., et al. 1999, A\&A, 349, L5

Hillier, D. J., \& Miller, D. L. 1999, ApJ, 519, 354

Hillier, D. J., \& Miller, D. L. 1998, ApJ, 496, 407

Jacob, R., Schönberner, D., \& Steffen, M. 2013, A\&A, 558, A78

Kaler, J. B., Stanghellini, L., \& Shaw, R. A. 1993, A\&A, 279, 529

Kwok, S., Purton, C. R., \& Fitzgerald, P. M. 1978, ApJ, 219, L125

Lodders, K. 2010, Astrophysics and Space Science Proceedings, 16, 379

Luridiana, V., Morisset, C., \& Shaw, R. A. 2015, A\&A, 573, A42

Medina, S., Peña, M., Morisset, C., et al. 2006, Rev. Mex. Astron. Astrofis., 42, 53

Miller Bertolami, M. M. 2016, A\&A, 588, A25

Miller Bertolami, M. M., \& Althaus, L. G. 2006, A\&A, 454, 845

Miszalski, B., Crowther, P. A., De Marco, O., et al. 2012, MNRAS, 423, 934

Montez, R., Kastner, J. H., Balick, B., et al. 2015, ApJ, 800, 8

Osterbrock, D. E., \& Ferland, G. J. 2006, Astrophysics of gaseous nebulae and active galactic nuclei

Peña, M., Stasińska, G., Esteban, C., et al. 1998, A\&A, 337, 866

Peña, M., Stasińska, G., \& Medina, S. 2001, A\&A, 367, 983

Peña, M., Medina, S., \& Stasińska, G. 2003, Revista Mexicana De Astronomia Y Astrofisica Conference Series, 38

Peña, M., Rechy-García, J. S., \& García-Rojas, J. 2013, Rev. Mex. Astron. Astrofis., 49, 87

Pottasch, S. R., Gathier, R., Gilra, D. P., et al. 1981, A\&A, 102, 237

Press, W. H., Teukolsky, S. A., Vetterling, W. T., et al. 1992, Cambridge: University Press

Raga, A. C., Riera, A., Mellema, G., et al. 2008, A\&A, 489, 1141

Ramos-Larios, G., Guerrero, M. A., Nigoche-Netro, A., et al. 2018, MNRAS, 475, 932

Ramos-Larios, G., \& Phillips, J. P. 2012, MNRAS, 425, 1091

Rechy-García, J. S., Peña, M., \& Velázquez, P. F. 2019, MNRAS, 482, 1163

Rechy-García, J. S., Velázquez, P. F., Peña, M., et al. 2017, MNRAS, 464, 2318

Sabbadin, F., Bianchini, A., \& Hamzaoglu, E. 1982, A\&AS, 50, 523

Sahai, R., Morris, M. R., \& Villar, G. G. 2011, AJ, 141, 134

Smith, L. F., \& Aller, L. H. 1969, ApJ, 157, 1245

Toalá, J. A., Ramos-Larios, G., Guerrero, M. A., et al. 2019, MNRAS, 485, 3360

Toalá, J. A., Guerrero, M. A., Todt, H., et al. 2015, ApJ, 799, 67

Todt, H., Sander, A., Hainich, R., et al. 2015, A\&A, 579, A75

Todt, H., Kniazev, A. Y., Gvaramadze, V. V., et al. 2013, MNRAS, 430, 2302
Todt, H., Peña, M., Hamann, W.-R., et al. 2013, 18th European White Dwarf Workshop., 303

Todt, H., Peña, M., Hamann, W.-R., et al. 2010, A\&A, 515, A83

Tody, D. 1993, Astronomical Data Analysis Software and Systems II, 173

Torres-Peimbert, S., \& Peimbert, M. 1977, Rev. Mex. Astron. Astrofis., 2, 181

Tylenda, R., Acker, A., \& Stenholm, B. 1993, A\&AS, 102, 595

Werner, K., Rauch, T., \& Kruk, J. W. 2007, A\&A, 474, 591

Zijlstra, A. A. 2015, Rev. Mex. Astron. Astrofis., 51, 221

99

MNRAS 000, 1-?? (2019) 